CALT-68-2386, CITUSC/02-019

HUTP-02/A018

hep-th/0205297

\title{
Worldsheet Derivation of a Large $N$ Duality
}

\author{
Hirosi Ooguri ${ }^{1}$ and Cumrun Vafa ${ }^{2}$ \\ ${ }^{1}$ California Institute of Technology 452-48, Pasadena, CA 91125, USA \\ 2 Jefferson Physical Laboratory, Harvard University, Cambridge, MA 02138, USA
}

\begin{abstract}
We give a worldsheet proof of the equivalence between the $U(N)$ Chern-Simons gauge theory on $S^{3}$ and the topological closed string theory on the resolved conifold geometry. When the 't Hooft coupling of the gauge theory is small, the dual closed string worldsheet develops a new branch. We show that the fluctuations of the worldsheet into this branch effectively correspond to "holes" on the worldsheet, generating an open string sector. This leads to a microscopic description of how the 't Hooft expansion of gauge theory amplitudes is reproduced in the closed string computation. We find that the closed string amplitudes also contain terms which are not captured in the 't Hooft expansion but are present in the exact computation in the gauge theory amplitudes. These arise when the whole Riemann surface is in the new branch. We also discuss the cases with $S O$ and $S p$ gauge groups.
\end{abstract}




\section{Introduction}

Many examples of large $N$ dualities have been discovered in string theory in recent years, in accord with 't Hooft's original idea [1]. Most of these results are motivated from the viewpoint of target space physics. However, the original intuition of ' $t$ Hooft was based on worldsheet diagrams. It is thus natural to expect that one can derive large $N$ dualities from the worldsheet perspective. In 't Hooft's double line notation, Feynman diagrams are expressed as surfaces with holes. It was postulated that somehow these holes get filled up, leading to closed Riemann surfaces without boundaries. Given that we know what the closed string duals are in many examples, it is natural to ask the reverse question. Namely, we could try to see how the holes get developed from the closed string perspective, leading to the Feynman diagrams of the gauge theories. In this paper, we show how this works in the context of the duality between the $U(N)$ Chern-Simons gauge theory on $S^{3}$ and the topological closed string theory on the resolved conifold, which was conjectured in [2]. By doing so, we also shed light on some aspects of this duality and in particular how certain non-perturbative terms in the gauge theory arise on the closed string dual. We suspect that our derivation may well have applications beyond the particular case considered here.

Let us first review the general setup of large $N$ dualities proposed by 't Hooft. Consider a gauge theory with $U(N)$ gauge group whose action is written as

$$
S=\frac{1}{g_{\mathrm{YM}}^{2}} \int \mathcal{L}(A)
$$

where $A$ is a gauge field. A Feynman diagram of $U(N)$ gauge theory drawn as a "ribbon graph" can be viewed as a closed Riemann surface with some holes. The dependence of the amplitude on the gauge coupling constant $g_{\mathrm{YM}}^{2}$ and the rank $N$ of the gauge group can be captured by the topology of this surface. For a ribbon graph with $g$ handles and $h$ holes, the amplitude comes with the factor

$$
\left(g_{\mathrm{YM}}^{2}\right)^{-V+E} N^{h}=\left(g_{\mathrm{YM}}^{2}\right)^{-V+E-h}\left(g_{\mathrm{YM}}^{2} N\right)^{h}=\left(g_{\mathrm{YM}}^{2}\right)^{2 g-2}\left(g_{\mathrm{YM}}^{2} N\right)^{h}=\left(g_{\mathrm{YM}}^{2}\right)^{2 g-2} t^{h},
$$

where $V$ and $E$ are the number of vertices and propagators in the graph, and we have defined $t=g_{\mathrm{YM}}^{2} N$. The full amplitude is given by summing over all topologies,

$$
F=\sum_{g=0}^{\infty} \sum_{h=1}^{\infty}\left(g_{\mathrm{YM}}^{2}\right)^{2 g-2} t^{h} F_{g, h}=\sum_{g}\left(g_{\mathrm{YM}}^{2}\right)^{2 g-2} F_{g}(t),
$$


where

$$
F_{g}(t)=\sum_{h=1}^{\infty} t^{h} F_{g, h}
$$

and the coefficients $F_{g, h}$ may be a function of other parameters defining the theory. The statement that this is an expansion in powers of $1 / N$ is the same as saying this is an expansion in powers of $g_{\mathrm{YM}}^{2}$, as shown in the above, since $g_{\mathrm{YM}}^{2} N$ is held fixed. The general conjecture of ' $\mathrm{t}$ Hooft is that at large $N$, with $g_{\mathrm{YM}}^{2} N=t$ held fixed, an equivalent description should involve closed Riemann surfaces which are obtained from the ribbon graphs by "filling holes with disks." Compare Figure 1 of a ribbon graph and Figure 2 of the corresponding Riemann surface:

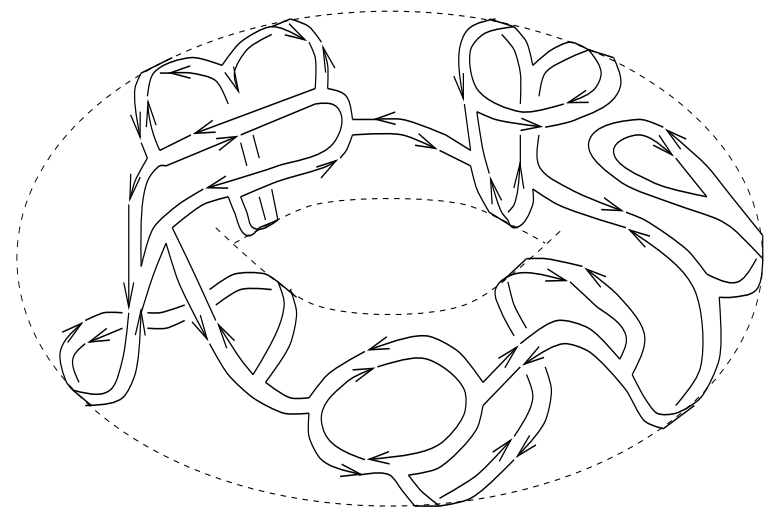

Figure 1: A Ribbon graph with $g=1$ and $h=9$.

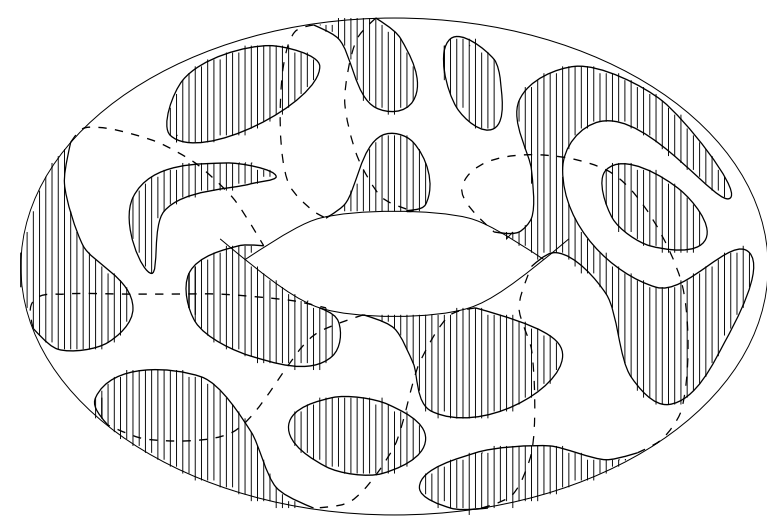

Figure 2: The corresponding Riemann surface. The holes in the ribbon graph in Figure 1 are filled with shaded regions, all of which have the topology of the disk.

In string theory, various $U(N)$ gauge theories can be realized on $\mathrm{D}$ branes. There, the ribbon graphs "come to life" as open string worldsheets with holes ending on D branes. In 
this setup, the string coupling $\lambda_{s}$ is identified with the gauge coupling as

$$
\lambda_{s}=g_{\mathrm{YM}}^{2}
$$

To interpret 't Hooft duality in the context of string theory, $F_{g}(t)$ in (1.1) should be viewed as a closed string genus $g$ amplitude which depends on some modulus in the target space given by $t=g_{\mathrm{YM}}^{2} N=\lambda_{s} N$.

Note that from the expression (1.2) for the 't Hooft expansion, one would conclude that $F_{g}(t)$ is a smooth analytic function of $t$ at $t=0$. However this raises a puzzle: Typically in large $N$ dualities, $t$ is interpreted as a size of a geometric object in the target space and $t \rightarrow 0$ leads to vanishing cycles, which in turn signal the breakdown of closed string perturbation theory and divergences of some amplitudes. On the other hand, from the dual gauge description, this lack of smoothness seems to be in conflict with the expectation that gauge theory perturbation should be reliable in this limit. This puzzle arises for any large $N$ dualities, including AdS/CFT correspondences. We will see how this puzzle is resolved in the case of the large $N$ duality for the Chern-Simons theory.

Since we believe our general strategy for a worldsheet derivation of large $N$ duality has a wider range of applicability, here we give its outline without specializing to the case of the Chern-Simons gauge theory. The basic outline of the idea was suggested in [2], and we will make it precise in the present paper.

That a worldsheet derivation could exist in string perturbation theory is natural since the closed string coupling constant $\lambda_{s}$ is kept small. On the other hand, $t=\lambda_{s} N$ is not necessarily taken to be small. This means that infinitely many holes can contribute to the amplitudes. This is very much like how we deform string backgrounds in closed string perturbation theory. There, we add marginal perturbations $t \int d^{2} z V(z, \bar{z})$ to the worldsheet action. We can compute worldsheet amplitudes by expanding it in powers of $t$, which counts the number of insertions of $V$ and we may find it necessary to take into account infinitely many insertions of $V$. Of course this is still compatible with string perturbation theory, which is an expansion in $\lambda_{s}$. That D-branes may be incorporated in such a manner in a closed string theory was also proposed in early days of D-branes [3].

The basic idea we shall use in a worldsheet derivation of the large $N$ duality is the following: We start with a "good" description of the worldsheet theory at $t=0$ from the perspective of the proposed closed string dual. By a "good" description we mean the one in which the worldsheet quantum field theory is well-defined and can be used to 
describe physics near $t=0$. In particular we assume that the expected breakdown of string perturbation theory and divergence of string amplitudes can be traced back to the emergence of an extra non-compact branch (or more specifically, the emergence of a noncompact region in the field space) in the otherwise perfectly well-defined quantum field theory in two dimensions. Such examples have already been encountered in singular limits of string compactification [4, 可, 6. Let us call the bulk of the field space by $H$ and the new emerging branch at $t=0$ by $C$. (The choice of the terminology is motivated by the fact that, in the Chern-Simons case, $H$ represents the Higgs branch and $C$ the Coulomb branch of the worldsheet theory.) Thus, when we integrate over field configurations on a fixed Riemann surface, there may be regions of the surface which are in the $H$ phase and there may be others in the $C$ phase. The $t$ dependence of the amplitudes will be captured entirely by the $C$ branch.

In principle, any configuration of $H$ and $C$ domains can contribute to the closed string amplitudes. However, in order to prove the correspondence between gauge theory and string theory, we need to establish that Riemann surfaces with mixed phases do not contribute to the topological string amplitudes, unless each connected component of the $C$ domain has the topology of the disk. This is to avoid configurations such as those shown in Figure 3, which do not correspond to ribbon graphs in the 't Hooft expansion:
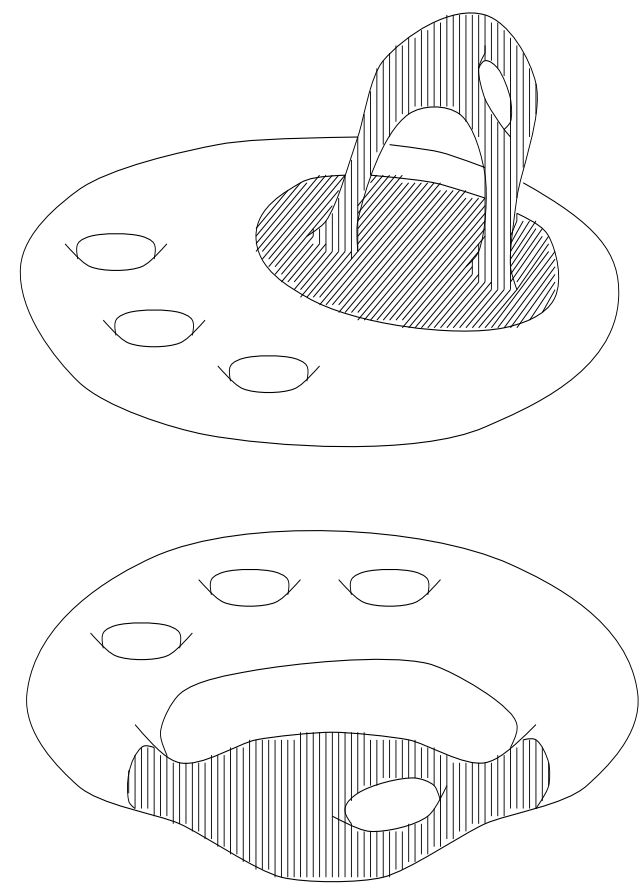


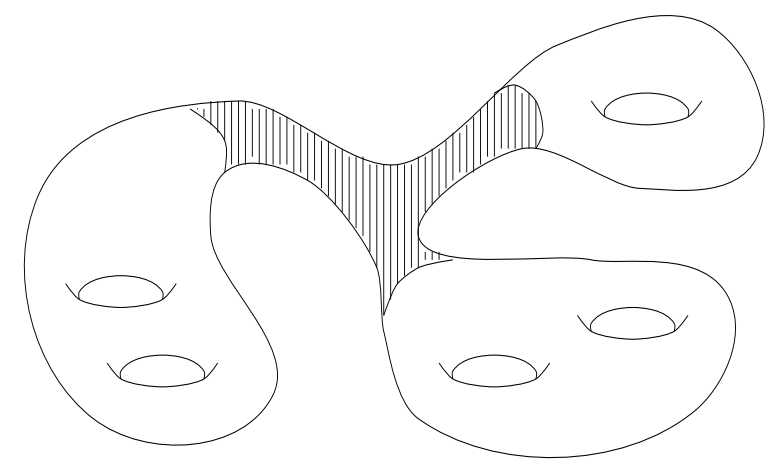

Figure 3: We need to show that worldsheets with both $C$ and $H$ phases, such as those shown here, do not contribute to the topological string amplitudes, except when every $C$ domain has the topology of the disk as shown in Figure 4. The $C$ domains are represented in the shaded regions in the figures.

Instead we show that the only mixed configurations which contribute to the topological string amplitudes are of the form in Figure 4:

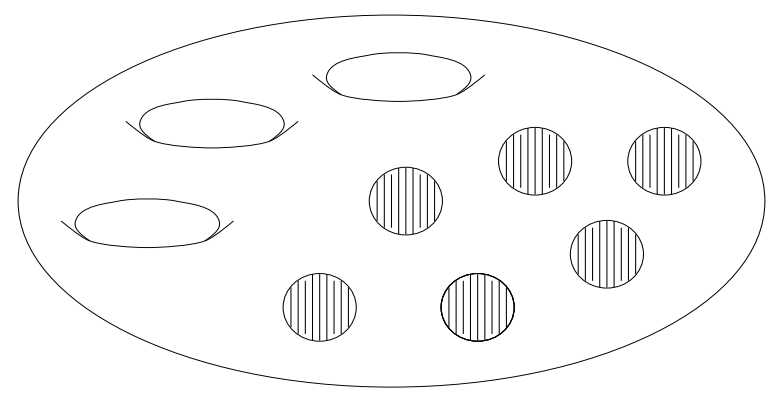

Figure 4: $A$ worldsheet with an arbitrary number of $C$ domains with the topology of the disk can contribute. We will show that each $C$ domain contributes with the factor of $t=g_{\mathrm{YM}}^{2} N$.

To reproduce the 't Hooft expansion (1.2), each $C$ domain of the disk topology should contribute by a factor of $t=g_{\mathrm{YM}}^{2} N$. Furthermore we need that the fields dynamical in the $H$ phase become non-dynamical in the $C$ phase (i.e. become infinitely massive in the IR in the $C$ phase), so that we obtain suitable D-brane like conditions imposed on the $H$ fields on the boundary. This precisely reproduces the structure of the 't Hooft expansion (1.1) of the gauge theory if we regard $C$ domains as holes of the ribbon graph, except that each of them gives rise to the necessary factor of $t$. To summarize, in this scenario, disks in the $C$ phase can be viewed as holes for some open string theory living in the $H$ phase. This will lead to a D-brane description and thus to a dual $U(N)$ gauge theory.

We have, however, two more possibilities: The full Riemann surface may be in the $H$ or $C$ branch. 
The appearance of a worldsheet in the pure $H$ phase is rather natural. If the whole Riemann surface is in the $H$ branch, we can consider it as contribution of purely closed string loops in the presence of D-branes before taking the decoupling limit. The fact that such contributions are included in our computation suggests that the large $N$ duality can be established in a more general sense. We believe that that our derivation should apply not only to the gauge theory/closed string duality in the strict low energy limit of string theory but also to a more general setup of the equivalence between a D-brane configuration and a closed string background, where the gravity is not decoupled in the D-brane side and the "near-horizon limit" is not taken in the closed string side. Of course if we take a decoupling limit, where the gauge theory decouples from gravity, the contribution from the entire Riemann surface being in the $H$ branch should vanish.

On the other hand, the case when the whole Riemann surface is in the $C$ branch, depicted in Figure 5 below, might appear as surprising from the gauge theory side, as it does not correspond to any perturbative 't Hooft diagram. This must thus correspond to some non-perturbative terms on the gauge theory side. The fact that the existence of $C$ branch corresponds to the breakdown of closed string amplitudes suggests that the contributions coming from Riemann surfaces which are entirely in the $C$ branch are not smooth in the limit $t \rightarrow 0$. From the perspective of gauge theory, this appears as the case where "the entire Riemann surface is a hole"! Thus our proof points out that the 't Hooft expansion of the gauge theory may miss important terms in the closed string side, which correspond to some non-perturbative terms in the gauge theory.

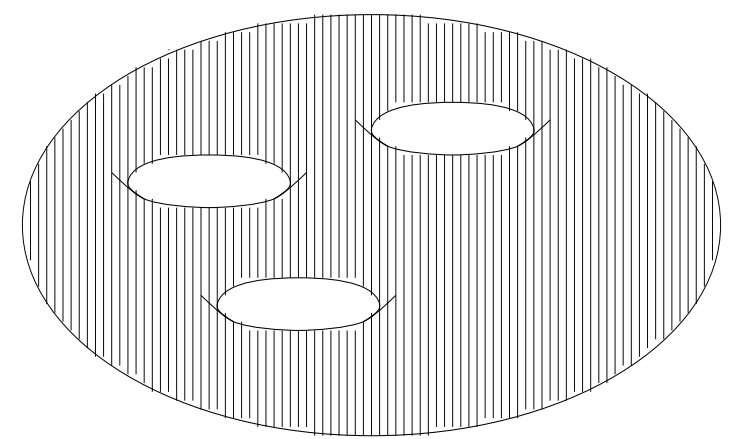

Figure 5: The entire worldsheet can be in the $C$ domain. This does not correspond to any perturbative 't Hooft diagram and therefore must represent some nonperturbative effect in the gauge theory.

In this paper we show how the above general ideas are realized in a precise quantitative manner for the large $N$ duality of Chern-Simons theory. The organization of this paper is 
as follows: In section 2 we review aspects of the Chern-Simons/topological closed string duality. In section 3 we review the linear sigma model description of the worldsheet theory on the resolved conifold. In section 4 we present the proof of the duality. We also give a brief discussion on the cases of the $S O(N)$ and $S p(N)$ gauge groups. We will conclude this paper in section 5 with summary of our results, generalization including Wilson loop observables, and discussion on implications of our results for other large $N$ dualities.

\section{Large $N$ Chern-Simons Duality}

The $U(N)$ Chern-Simons gauge theory at level $k$ on $S^{3}$ was conjectured in [2] to be equivalent to topological closed strings on resolved conifold, with the dictionary that the string coupling constant is given by $\lambda_{s}=i /(k+N)$ and the complexified Kähler class $t$ of the resolved conifold is $t=i N /(k+N)$. Since $t$ is pure imaginary, the $S^{2}$ of the conifold geometry has zero size but there is a non-vanishing NS-NS two-form field, which makes the string theory non-singular. We will often refer to $t$ as the size of the $S^{2}$. The motivation for the conjecture comes from the fact that both sides can be viewed as topological strings near the conifold. The $U(N)$ Chern-Simons theory can be viewed as target string field theory for topological A-model on the deformed conifold where there are $N$ D-branes wrapping around $S^{3}$ [7]. The gravity dual is the topological string on the resolved conifold. Thus, very much in the spirit of large $N$ dualities, there is a topological transition where the $S^{3}$ cycle containing D-branes shrinks and there emerges a dual cycle $S^{2}$. In fact, since

the Chern-Simons theory is topological, the partition function does not depend on the volume of the $S^{3}$, so we can take the $S^{3}$ size to zero. On the other hand, since $t$ is pure imaginary, the resolved conifold geometry in the closed string side is also at zero size. So, geometrically speaking, the duality is the conversion of branes to NS-NS $B$-field, as shown in Figure 6 below, without touching the underlying geometry. 


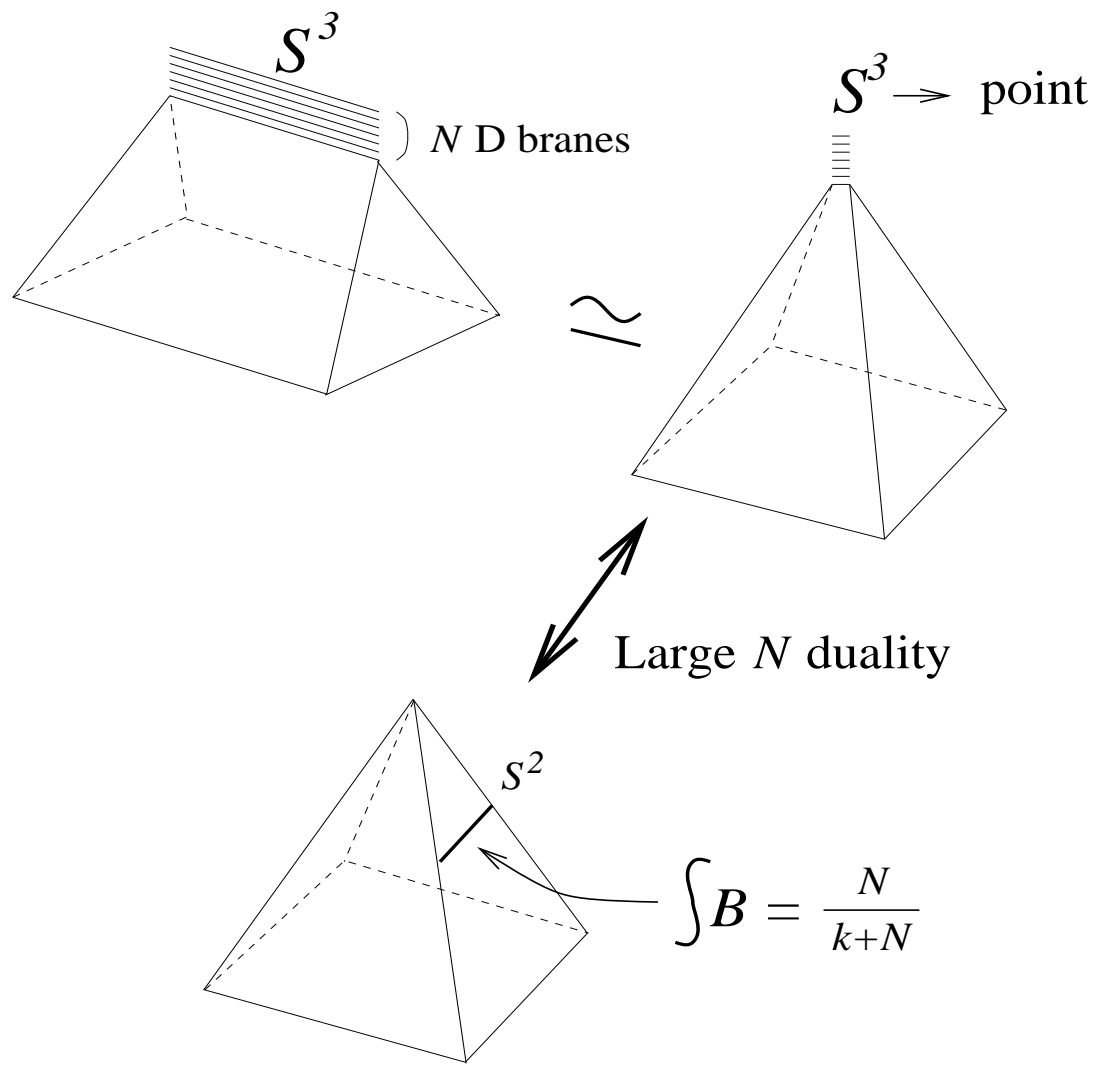

Figure 6: The Chern-Simons theory can be realized as the topological open string theory on $D$ branes wrapping on the $S^{3}$ of the deformed conifold. Topological string amplitudes are independent of the size of the $S^{3}$, so we can shrink it to zero. Thus the large $N$ duality is the statement that $N D$ branes on the conifold can be replaced by the $B$-field of the amount $g_{\mathrm{YM}}^{2} N$.

Many checks have been performed for this conjectured duality including highly nontrivial tests involving Wilson loop operators to all order in the gauge coupling constant [8,9,[10,11].1 It is clearly desirable to go beyond gathering evidence for large $N$ dualities and actually prove them. The Chern-Simons theory/topological closed string duality was embedded in the context of the type IIA string theory in [13]. It is known [8] that amplitudes of the Chern-Simons theory can be used to compute the $F$ terms in the $\mathcal{N}=1$ gauge theory which is realized on $N$ D6 branes wrapping around the $S^{3}$ in the deformed conifold geometry. On the other hand, topological closed string amplitudes give corresponding terms in the low energy effective theory of the type IIA string theory on the resolved conifold with $N$ units of the Ramond-Ramond flux through the $S^{2}$ [14]. Therefore we have

1 The original conjecture was for the gauge group $S U(N)$, but it was pointed out in [12] that the duality can be stated in a more natural form in the context of $U(N)$ gauge theory. 
a proof of the Chern-Simons/topological closed string duality provided one can show that the deformed conifold with D6 branes and the resolved conifold without brane are related in the type IIA string theory. Subsequently it was found that these two configurations are indeed smoothly connected to each other by lifting them to $M$ theory on a $G_{2}$ holonomy manifold [15]. The existence and absence of D-branes on these two configurations are traced to the fact that, depending on the moduli of the $G_{2}$ manifold, a $Z_{N}$ action on the $G_{2}$ manifold does or does not have a fixed point on the $S^{3}$. Moreover one can show that the $F$ terms in question do not depend on these moduli. Therefore the $M$ theory duality implies the Chern-Simons/topological closed string duality. Various aspects of the lifting of type IIA configurations to $G_{2}$ holonomy manifolds have been studied in a number of papers [16, 17, 18].

This derivation is fine, except that one is appealing to the duality between the type IIA theory and $M$ theory compactified on a circle, which remains a conjecture. The aim of this paper is to give a self-contained proof of the gauge theory/string theory duality without making use of the $M$ theory/type IIA duality. The $g$ loop topological string amplitude is evaluated by computing the functional integral of the sigma-model for the resolved conifold and then integrating the resulting amplitude over the moduli space of genus $g$ Riemann surface. We will show explicitly that this string amplitude can be expressed as a sum of Feynman diagrams in the Chern-Simons gauge theory.

That there may exist a worldsheet derivation of the Chern-Simons/ topological strings was suggested in [2]. The basic idea advocated there was to start from the closed string side and go to a regime where the conifold geometry is singular, which corresponds to small 't Hooft coupling in the gauge theory side. In this limit, the worldsheet theory acquires Coulomb and Higgs phases, which can co-exist on a single worldsheet. The basic proposal there was that the regions on the worldsheet which are in the Coulomb phase become the holes from the perspective of the Higgs phase and that this effectively introduces an open string sector in the string theory. In this paper, we show that this general idea is correct and prove that amplitudes in the Chern-Simons theory are quantitatively reproduced in the topological closed string computation.

For strings on the resolved conifold, where various aspects of the worldsheet dynamics have been studied [19,6], it is known that the spectrum becomes continous without mass gap and that $g$ loop topological string amplitudes diverges as $1 / t^{2 g-2}$ in the limit $t \rightarrow 0$. This is in line with the expectation that string theory perturbation breaks down in this 
limit. However, as discussed in the introduction, this seems to be at odds with the structure of the 't Hooft expansion of $F_{g}(t)$ shown in (1.2) and its smoothness as $t \rightarrow 0$.

It turns out that the exact Chern-Simons amplitudes also contain terms singular in $t$. That these amplitudes have such extra terms that are missing in the perturbative expansion has been noted and how they appear from the path-integral has been studied in [20] and 21,22,23]. Similar non-perturbative terms arise in the context of the emedding of this duality in type IIA superstrings. It is important for our derivation of large $N$ dualities to study these extra terms which are not captured by the ' $t$ Hooft expansion.

\subsection{Nonperturbative terms; Chern-Simons perspective}

Let us first discuss the appearance of these non-perturbative terms from the perspective of the Chern-Simons gauge theory [20,21,22,23]. The idea is to consider the prefactors that go into the definition of the path integral. The $U(N)$ Chern-Simons perturbation theoy on $S^{3}$ expands the gauge field near the trivial connection $A=0$. This connection has a residual global gauge symmetry corresponding to constant $U(N)$ gauge transformations. This implies that the path-integral must have an extra factor of $1 / \operatorname{vol}_{\mathrm{CS}}(U(N))$, where the volume vol $_{C S}$ is to be measured using the normalization defined by the quadratic term in the Chern-Simons action. Since this factor is $k / 2 \pi$, and it is quantum corrected to $(k+N) / 2 \pi$, this means that the radius of $U(N)$ is not 1 , but rather $\sqrt{(k+N) / 2 \pi}$. Thus, rescaling the radius to 1 , and denoting the corresponding volume by $\operatorname{vol}(U(N))$, we see that the path integral should have a prefactor

$$
\exp (\mathcal{F})=\frac{(2 \pi i)^{\frac{1}{2} \operatorname{dim}(U(N))}}{\left(\frac{k+N}{2 \pi}\right)^{\frac{1}{2} \operatorname{dim}(U(N))} \operatorname{vol}(U(N))} \exp \left(\mathcal{F}_{\text {pert. }}\right),
$$

where $\mathcal{F}_{\text {pert }}$ is computable by summing over the 't Hooft diagrams. The factor $(2 \pi i)^{\frac{1}{2} \operatorname{dim}(U(N))}$ in the numerator comes from the Gaussian integral formula $\int d x e^{-\frac{1}{2 i} x^{2}}=$ $\sqrt{2 \pi i}$.

The non-perturbative piece of the amplitude in this case is then given by

$$
\mathcal{F}_{\text {nonpert }}=-\log (\operatorname{vol}(U(N)))-\frac{1}{2} \operatorname{dim}(U(N)) \log \left(\frac{k+N}{(2 \pi)^{2} i}\right) .
$$

Let us evaluate this formula. Since $U(N)=U(1) \otimes S U(N) / Z_{N}$ and since vol $U(1)=2 \pi \sqrt{N}$ in our normalization of the kinetic term, we have

$$
\operatorname{vol}(U(N))=\frac{2 \pi}{\sqrt{N}} \operatorname{vol}(S U(N))
$$


The volume of a compact group has been computed in [24]. Applying this formula to $S U(N)$, one obtains

$$
\operatorname{vol}(S U(N))=\operatorname{vol}\left(\mathrm{g} / \mathrm{g}_{\mathbf{z}}\right) \cdot \operatorname{vol}\left(S^{3} \times S^{5} \times \cdots S^{2 N-1}\right),
$$

where $\mathrm{g}$ is the Lie algebra of $U(N), \mathrm{g}_{\mathbf{Z}}$ is the Chevalley lattice, and $S^{2 i-1}(i=2,3, \cdots, N)$ are unit spheres with dimensions $(2 i-1)$. The odd dimensional spheres appear in the volume formula since $S U(N)$ is rational homotopy equivalent to the product of these spheres. Evaluating this formula, we find2

$$
\operatorname{vol}(S U(N))=\frac{\sqrt{N}(2 \pi)^{\frac{1}{2} N^{2}+\frac{1}{2} N-1}}{(N-1) !(N-2) ! \cdots 2 ! 1 !}=\frac{\sqrt{N}(2 \pi)^{\frac{1}{2} N^{2}+\frac{1}{2} N-1}}{G_{2}(N+1)}
$$

where $G_{2}(z)$ is the Barnes function defined by

$$
G_{2}(z+1)=\Gamma(z) G_{2}(z), \quad G_{2}(1)=1 .
$$

Combining this with (2.3), we find

$$
\operatorname{vol}(U(N))=\frac{(2 \pi)^{\frac{1}{2} N^{2}+\frac{1}{2} N}}{G_{2}(N+1)}
$$

Substituting this into (2.1), we obtain the nonperturbative piece of the Chern-Simons amplitude as

$$
\mathcal{F}_{\text {nonpert }}=\log \left(e^{\frac{\pi}{8} i N^{2}}\left(\frac{2 \pi}{k+N}\right)^{\frac{1}{2} N^{2}} \frac{G_{2}(N+1)}{(2 \pi)^{\frac{1}{2} N}}\right) .
$$

This precisely reproduces the $k \rightarrow \infty$ limit of the exact result [20] in the Chern-Simons theory.

Now let us take the large $N$ expansion of this formula. Using the Binet integral for the Gamma function

$$
\log \Gamma(z)=\left(z-\frac{1}{2}\right) \log z-z+\frac{1}{2} \log 2 \pi+2 \int_{0}^{\infty} \frac{\tan \left(\frac{t}{z}\right)}{e^{2 \pi t}-1} d t,
$$

2 Since the kinetic term of the Chern-Simons theory uses the trace in the fundamental representation, the normalization of the metric on $S U(N)$ differs by a factor $\sqrt{2 N}$ from that in the mathematical literature. We thank Y. Hashimoto for useful communication and for having his unpublished notes available for us. 
one can derive the asymptotic expansion of the Barnes function as

$$
\begin{aligned}
\log G_{2}(N+1)= & \frac{N^{2}}{2} \log N-\frac{1}{12} \log N-\frac{3}{4} N^{2}+\frac{1}{2} N \log 2 \pi+\zeta^{\prime}(-1) \\
& +\sum_{g=2}^{\infty} \frac{B_{2 g}}{2 g(2 g-2) N^{2 g-2}}
\end{aligned}
$$

Recognizing $(k+N)=1 / \lambda_{s}$, we obtain

$$
\begin{aligned}
\mathcal{F}_{\text {nonpert }}=\frac{N^{2}}{2} & \left(\log \left(2 \pi i N \lambda_{s}\right)-\frac{3}{2}\right)-\frac{1}{12} \log (N)+\zeta^{\prime}(-1) \\
& +\sum_{g=2}^{\infty} \frac{1}{N^{2 g-2}} \frac{B_{2 g}}{2 g(2 g-2)},
\end{aligned}
$$

which we can rewrite in terms of $\lambda_{s}$ and $t=N \lambda_{s}$ as

$$
\begin{gathered}
\mathcal{F}_{\text {nonpert }}=\frac{1}{2} \lambda^{-2} t^{2}\left(\log (2 \pi i t)-\frac{3}{2}\right)-\frac{1}{12} \log \left(t \lambda_{s}^{-1}\right)+\zeta^{\prime}(-1) \\
+\sum_{g=2}^{\infty} \lambda_{s}^{2 g-2} \frac{B_{2 g}}{2 g(2 g-2) t^{2 g-2}} .
\end{gathered}
$$

As we will show in section 4.2, this structure of singularity predicted by the prefactors in Chern-Simons theory exactly agrees with the result of the topological closed string computation for worldsheets in the pure $C$ phase. 3

\subsection{Nonperturbative terms; type IIA superstring perspective}

These nonperturbative terms also have physical interpretations in the context of the type IIA string theory, which is far more non-trivial. The Chern-Simons amplitude compute some $F$-terms in the $\mathcal{N}=1$ gauge theory realized on D6 branes wrapping on $S^{3}$ on the deformed conifold [14]. In particular the planar diagrams of the Chern-Simons theory compute superpotential terms in the gauge theory involving the gaugino bilinear superfield $S=\operatorname{Tr} \psi^{2}$. If $F_{0, h}$ denote the Chern-Simons amplitude with worldsheet topology of genus 0 and with $h$ holes, one obtains a superpotential

$$
W_{\text {pert }}(S)=N \sum_{h=1}^{\infty} F_{0, h} S^{h-1}=N \sum_{n \neq 0}(S+i n) \log (S+i n)
$$

3 In order to check this, one has to recall the ambiguities inherent in the definition of closed string partition function genus 0 and 1 . 
On the other hand, the exact answer for the Chern-Simons gauge theory is known, and the leading term in the large $N$ expansion yields

$$
W_{\text {exact }}(S)=N \sum_{n}(S+i n) \log (S+i n)=N \frac{\partial F_{0}}{\partial S}
$$

where $F_{0}$ is the genus 0 closed string amplitude on the resolved conifold with Kahler class $t=S$. Compared with (2.13), the sum here includes the $n=0$ term, which corresponds to $N S \log S$ term. This term is absent from the perturbative expression (2.13) but is a part of the exact Chern-Simons amplitude, as discussed above.

The splitting of the exact Chern-Simons partition function (2.14) to perturbative and non-perturbative pieces has a physical interpretation in type IIA superstring. The low energy effective theory of $N$ D6 branes wrapping $S^{3}$ is the $\mathcal{N}=1$ Yang-Mill theory. The superpotential $W(S)$ receives stringy corrections, and the perturbative string computation is caputured in $W_{\text {pert }}(S)$. However, there are also non-perturbative contributions: Gauge theory instantons generate a superpotential for $S$. In fact to leading order in IR, which means the small $S$ regime, the measure induced potential $N S \log S$ [25] and the $\tau S$ tree term, with $\tau=g_{\mathrm{YM}}^{-2}$, dominate the superpotential and lead to gaugino condensate

$$
d W=0 \rightarrow d(N S \log S+\tau S)=0 \rightarrow S \sim e^{-\tau / N}
$$

Thus it is not surprising that $S \log S$ is missing from the pertubation theory: it should come from axial anomaly in path integral measure. That they are related to fractional instantons is reflected in the fact that the condensate is exponential $\tau / N$ which is $1 / N$-th of the action for an instanton. The reason that this survives at large $N$ is precisely because they are fractional, so that they are weighted with $1 / N$ and one gets the action as inverse of 't Hooft parameter $1 / N g_{\mathrm{YM}}^{2}=1 / t$.

Note that the wrapping Euclidean D2 brane, which from the viewpoint of D6 brane is an instanton, is not an instanton from the viepoint of the Chern-Simons gauge theory since it is not localized to a point on $S^{3}$. This is consistent with the fact that these nonperturbative terms appear in the prefactor $1 / \operatorname{vol}(U(N))$ in the context of Chern-Simons gauge theory.

Similar contributions exists at each order in $1 / N$. As discussed in [13], each term in the $1 / N$ expansion correspond to some contribution to a superpotential-type term. Moreover at each order in the $1 / N$ expansion, which corresponds to a fixed genus on the closed string dual, the superpotential terms can be viewed as contributions from perturbative 
string expansions plus one term at each genus which can be presumably attributed to the contribution of fractional instantons to the corresponding amplitudes. If $F_{g}(S)$ denotes the dual closed string amplitude at genus $g$ the leading term as $S \rightarrow 0$ is precisely the term missing from open string/gauge theory perturbations. For $g \geq 2$, it is given by

$$
F_{g}(S) \rightarrow \frac{B_{2 g}}{2 g(2 g-2)} S^{2-2 g} \quad(S \rightarrow 0) .
$$

We will show that, in the topological closed string side, these nonperturbative terms correspond to the situation in which the entire worldsheet is in the Coulomb phase, i.e. when the worldsheet as a whole is a "hole." This configuration does not correspond to any Feynman diagram of the gauge theory, and it explains why these terms are not captured in the perturbative gauge theory computation. Thus, both the exact answer in Chern-Simons theory and closed string dual have these extra terms, and this is perfectly consistent with the large $N$ duality.

\section{Linear sigma-model description of the worldsheet}

One of the main difficulties in finding a direct proof of the $A d S / C F T$ correspondence has been a lack of a useful description of string worldsheet when the 't Hooft coupling in the gauge theory side is small. In fact, for string theory on the resolved conifold, there is such a description. The linear sigma-model [26] is a good description for the worldsheet even when the size $t$ of the base $S^{2}$ is small.

Before describing the linear sigma-model, it would be useful to start with a brief review of the conifold geometry. The conifold is a singular space defined by the equation,

$$
\sum_{i=1}^{4} z_{i}^{2}=0
$$

in $\mathbf{C}^{4}$. The Kähler form is given by

$$
\omega=\frac{1}{2 i} \sum_{i=1}^{4} d z_{i} \wedge d \bar{z}_{i}
$$

restricted on (3.1). The space has a singularity at $z_{i}=0$, and it can be made smooth either by deformation of complex structure or by small resolution. The deformation of the conifold is described by the equation,

$$
\sum_{i=1}^{4} z_{i}^{2}=\mu .
$$


Without loss of generality, we can assume that $\mu$ is real and positive. The resulting space is nothing but the cotangent space of $S^{3}$. To see this, we set

$$
z_{i}=x_{i}+i p_{i}
$$

The equation can then be expressed as

$$
\sum_{i} x_{i}^{2}-\sum_{i} p_{i}^{2}=\mu, \quad \sum_{i} x_{i} p_{i}=0
$$

The first equation shows that the space contains an $S^{3}$ of radius $\mu$ at $p_{i}=0$, and the second equation means that $p_{i}$ 's are coordinates of the cotangent space at $x \in S^{3}$. Moreover the Kähler form (3.2) is expressed in these coordinates as

$$
\omega=\sum_{i=1}^{4} d p_{i} \wedge d x_{i}
$$

which gives the natural symplectic structure on $T^{*} S^{3}$. Thus the space (3.3) with $\mu \neq 0$ is smooth deformation of the singular space (3.1).

The conifold can also be made smooth by small resolution. It means finding a smooth space which can be mapped holomorphically onto the conifold (3.1) except at the singularity at $z_{i}=0$. Thus it is not a complex structure deformation but should be considered as deformation of the Kähler structure. To describe the process, it is useful to introduce a new set of complex coordinates $y_{i}(i=1, \cdots, 4)$ defined by

$$
\begin{aligned}
& y_{1}=z_{1}+i z_{2}, \quad y_{2}=z_{1}-i z_{2}, \\
& y_{3}=z_{3}+i z_{4}, \quad y_{4}=-z_{3}+i z_{4} .
\end{aligned}
$$

The conifold equation (3.1) can then be written as

$$
y_{1} y_{2}-y_{3} y_{4}=0
$$

Introducing the two sets of coordinates $\left(y_{1}, y_{2}, y_{3}, z\right)$ and $\left(w, y_{2}, y_{3}, y_{4}\right)$ defined by

$$
\begin{aligned}
\left(y_{1}, y_{2}, y_{3}, y_{4}\right) & =\left(y_{1}, y_{2}, y_{3}, z y_{1}\right) \\
& =\left(w y_{4}, y_{2}, y_{3}, y_{4}\right)
\end{aligned}
$$

the equation (3.8) becomes

$$
y_{1}\left(y_{2}-z y_{3}\right)=0
$$


in the $\left(y_{1}, y_{2}, y_{3}, z\right)$ coordinates and

$$
y_{4}\left(w y_{2}-y_{3}\right)=0
$$

in the $\left(y_{2}, y_{3}, y_{4}, w\right)$ coordinates. This suggests considering two smooth spaces

$$
U_{1}=\left\{\left(y_{1}, y_{2}, y_{3}, z\right) \mid z y_{2}-y_{3}=0\right\}, \quad U_{2}=\left\{\left(y_{2}, y_{3}, y_{4}, w\right) \mid y_{2}-w y_{3}=0\right\},
$$

glued together by $z w=1$ and $y_{1}=z y_{4}$. The resulting space is smooth, and it is mapped onto the conifold (3.8) by (3.9). The map is holomorphic except at $y_{i}=0$, and this gives the small resolution.

The inverse image of the singularity $y_{i}=0$ is $z$ and $w$ glued together by $z w=1$, namely we can regard $z$ and $w$ as coordinates on the northern and the southern hemispheres of $S^{2}$. In this way, the conifold singularity is resolved by replacing the singularity at $y_{i}=0$ by the $S^{2}$. The equations $y_{4}=z y_{1}$ and $y_{2}=z y_{3}$ define a sum of line bundles $\mathcal{O}(-1) \oplus \mathcal{O}(-1)$ over the $S^{2}$, where $\left(y_{1}, y_{3}\right)$ are regarded as coordinates of the fiber over the northern hemisphere and $\left(y_{2}, y_{4}\right)$ are over the southern hemisphere of the $S^{2}$.

To construct a sigma-model whose target space is the resolved conifold, it is convenient to use the projective coordinates $\left(a_{1}, a_{2}\right)$ on the $S^{2}$ so that $z=a_{1} / a_{2}$. The north pole is at $a_{1}=0$ and the south pole is at $a_{2}=0$. The coordinates $y_{i}$ 's can then be expressed as

$$
\begin{aligned}
& y_{1}=a_{1} b_{1}, \quad y_{2}=a_{2} b_{2}, \\
& y_{3}=a_{1} b_{2}, \quad y_{4}=a_{2} b_{1} .
\end{aligned}
$$

In these coordinates, the $S^{2}$ is located at $b_{1}=b_{2}=0$. The variables $a_{i}, b_{i}$ are arbitrary, but there is a gauge symmetry

$$
\left(a_{i}, b_{i}\right) \rightarrow\left(\zeta a_{i}, \zeta^{-1} b_{i}\right), \quad \zeta \in \mathbf{C} \backslash\{0\},
$$

which keeps $y_{i}$ 's invariant. Thus the resolved conifold can be constructed as the Kähler quotient of $\left(a_{1}, a_{2}, b_{1}, b_{2}\right)$ by the action of (3.14).

This geometric construction is translated into the field theory language as follows. We consider a linear sigma-model in two dimensions with $\mathcal{N}=2$ supersymmetry consisting of four chiral multiplets, $A_{i}, B_{i}(i=1,2)$, whose lowest components are $a_{i}, b_{i}$ in the above paragraph, and one vector multiplet $V$ for the $U(1)$ gauge symmetry to enforce the gauge symmetry (3.14). The four charged chiral fields carry $U(1)$ charges +1 and -1 for $A$ and $B$ respectively. 
Let us look at the potential for the scalar fields. The kinetic terms $\bar{A}_{i} e^{2 V} A_{i}+\bar{B}_{i} e^{-2 V} B_{i}$ gives

$$
V_{k i n}=2|\sigma|^{2}\left(\left|a_{1}\right|^{2}+\left|a_{2}\right|^{2}+\left|b_{1}\right|^{2}+\left|b_{2}\right|^{2}\right)-D\left(\left|a_{1}\right|^{2}+\left|a_{2}\right|^{2}-\left|b_{1}\right|^{2}-\left|b_{2}\right|^{2}\right)
$$

where $e$ is the $U(1)$ gauge coupling constant on the worldsheet, $\sigma$ is the complex scalar field, and $D$ is the auxiliarly field in the vector multiplet $V$. Note that both $e$ and $\sigma$ have dimensions of mass on the worldsheet. The gauge kinetic energy contributes $\frac{-1}{2 e^{2}} D^{2}$. In addition, we can add the Fayet-Illiopoulos term $-r D$ and the theta term $i \frac{\theta}{2 \pi} F$ for the gauge field strength $F$. Eliminating the auxiliarly field $D$ by its equation of motion, we obtain

$$
V=2|\sigma|^{2}\left(\left|a_{1}\right|^{2}+\left|a_{2}\right|^{2}+\left|b_{1}\right|^{2}+\left|b_{2}\right|^{2}\right)+\frac{e^{2}}{2}\left(\left|a_{1}\right|^{2}+\left|a_{2}\right|^{2}-\left|b_{1}\right|^{2}-\left|b_{2}\right|^{2}-r\right)^{2} .
$$

If $r \neq 0$, solutions to $V=0$ are given by $\sigma=0$ and $a_{i}, b_{i}$ obeying the constraint

$$
\left|a_{1}\right|^{2}+\left|a_{2}\right|^{2}-\left|b_{1}\right|^{2}-\left|b_{2}\right|^{2}=r
$$

modulo the gauge symmetry

$$
a_{i} \rightarrow e^{i \theta} a_{i}, b_{i} \rightarrow e^{-i \theta} b_{i}
$$

The resulting space is the resolved conifold. To see this, suppose $r>0$. (For $r<0$, one can just exchange the roles of $a_{i}$ and $b_{i}$ below.) The equation (3.17) says that the space contains a minimum $S^{2}$ of radius $\sqrt{r}$ at $b_{i}=0$. Because of (3.13), this $S^{2}$ is located at $y_{i}=0$, the location of the singularity of the conifold. The gauge symmetry (3.14) is partially fixed by (3.17), with the residual gauge symmetry manifest in (3.18).

When $r=0$, the space of solutions to $V=0$ acquires an additional branch where $\sigma \neq 0$ and $a_{i}, b_{i}=0$ [26]. If $\theta \neq 0$, the theta term induces a constant electric field [27],

$$
F=\frac{e^{2}}{2 \pi} \tilde{\theta}
$$

where $\tilde{\theta} \equiv \theta(\bmod 2 \pi \mathbf{Z})$ and $|\tilde{\theta}|<\pi$. This gives rise to the vacuum energy density

$$
E_{\text {vacuum }}=\frac{e^{2}}{2}\left(\frac{\tilde{\theta}}{2 \pi}\right)^{2}
$$


for this branch. In addition, if $r \neq 0$, it gives an additional contribution of $\frac{e^{2}}{2} r^{2}$ when $\sigma$ is large. (This is obtained by setting $a_{i}, b_{i}=0$ in (3.16).) The vaccum energy density for large $\sigma$ can therefore be expressed as

$$
E_{\text {vacuum }}=\frac{e^{2}}{2}\left[r^{2}+\left(\frac{\tilde{\theta}}{2 \pi}\right)^{2}\right]
$$

Thus, in the limit $r \rightarrow 0$ and $\theta \rightarrow 0(\bmod 2 \pi \mathbf{Z})$, this branch is degenerate with the branch with $\sigma=0$.

Classically, in this limit we would expect two branches: the Coulomb branch $C$ where $|\sigma| \neq 0$ which implies that $a_{i}, b_{i}$ are massive and the Higgs branch where $|\sigma|=0$ but the $a_{i}, b_{i}$ are not zero. In the Higgs branch, $\sigma$ field is massive and the $U(1)$ gauge symmetry is spontaneously broken. It is known that, in the quantum theory these two branches are smoothly connected. In the infrared limit, one expects that, even though the two branches are still connected, the region that interpolates between them stretches out infinitely long. For finite $e^{2}$, wave functions can spread from the Higgs branch to the Coulomb branch. However the distance between these branch becomes infinite in the IR limit $e^{2} \rightarrow \infty$ and thus the two branches decouple. It is believed that the Higgs branch theory is obtained by the straightforward $e^{2} \rightarrow \infty$ limit of the linear sigma model, whereas the decoupled Coulomb branch theory is obtained by keeping the combination $\widetilde{\sigma}=|e|^{-1} \sigma$ finite in taking the limit and by performing the functional integral over $\widetilde{\sigma}$ rather than over $\sigma$ [28,29]. This Coulomb branch being disconnected from the Higgs branch plays no role in this paper. In the following, we will focus on the Higgs branch theory since its infrared limit is the non-linear sigma-model on the resolved conifold. However, in the Higgs branch, there is a region where one connects to the Coulomb branch. This is the infinitely long throat which used to be a transition region to the Coulomb branch. This throat region, where $|\sigma| \gg 1$, can be viewed as a "Coulomb domain" of the Higgs branch. We will refer to it as the $C$ domain or $C$ branch. This should not cause any confusion as the IR disconnected Coulomb branch plays no role in the string theory studied in this paper.

We will find it useful to note that the dependence of the linear sigma-model action on $r$ and $\theta$ can be expressed as a superpotential

$$
W=t \Sigma
$$

where

$$
t=\frac{\theta}{2 \pi}+i r
$$


and $\Sigma$ is a twisted chiral field defined as the field strength of the vector multiplet,

$$
\Sigma=\bar{D}_{+} D_{-} V
$$

In this paper, we are interested in the $A$-type topological twist of the sigma-model. Field configurations invariant under the topological BRST symmetry are given by holomorphic maps from the worldsheet to the resolved conifold, i.e., worldsheet instantons. One can show [26] that the instanton actions for such configurations are given by

$$
S_{\text {instanton }}=-2 \pi i t N
$$

where $N$ is the instanton number. Thus we can identity $t$ as the complexified Kähler modulus of the resolved conifold. When $t$ is non-zero (with $\operatorname{Im} t \geq 0$ ), topological string amplitudes can be expresed as a sum over worldsheet instantons weighted with the factor $e^{2 \pi i t N}$. As we take the conifold limit $t \rightarrow 0$, the sum over the instantons may diverge. In the linear sigma-model description, the divergence occurs since the non-compact branch with $\sigma \neq 0$ opens up in the limit.

\section{Derivation of the large $N$ duality}

In this section, we will present a detailed derivation of the equivalence between the Chern-Simons gauge theory on $S^{3}$ and the topological closed string on the resolved conifold.

To compute a topological string amplitude, one starts with the topological A model, i.e. the A-type topological twist of the linear sigma-model described in section 2, evaluate a certain correlation function of the model, and integrate it over the moduli space of Riemann surfaces. For example, the $g$ loop topological string partition function is given by논

$$
\mathcal{F}_{g}=\int_{\mathcal{M}_{g}}\left\langle\prod_{i=1}^{3 g-3}\left(\eta_{i}, G_{L}^{-}\right)\left(\bar{\eta}_{i}, G_{R}^{-}\right)\right\rangle,
$$

where $\mathcal{M}_{g}$ is the moduli space of genus- $g$ Riemann surfaces, $G_{L}^{-}$and $G_{R}^{-}$are $\mathcal{N}=2$ supercurrents which have conformal dimensions $(2,0)$ and $(0,2)$ after the topological twist, $\eta_{i}$ and $\bar{\eta}_{i}$ are Beltrami differentials on the Riemann surface $\mathcal{S}$, and

$$
\left(\eta_{i}, G_{L}^{-}\right)=\int_{\mathcal{S}} d z^{2} \eta_{i}(z) G_{L}^{-}(z)
$$

4 We are using the symbol $\mathcal{F}_{g}$ to denote the string amplitude, to distinguish it from the corresponding gauge theory amplitude $F_{g}$. 
When the Kähler modulus $t$ of the resolved conifold is large, we can express the topological string amplitude as a sum of worldsheet instantons, i.e., a sum of holomorphic maps from genus $g$ surface to the resolved conifold. We will show that, when $t$ is small, the same amplitude can be expressed as an asymptotic expansion in powers of $t$, and each term in the expansion can be idenfitied as a sum over Feynman digrams of the Chern-Simons gauge theory drawn as genus $g$ ribbon graphs with a fixed number of holes.

As we saw in (3.21), when the complex scalar field $\sigma$ in the vector multiplet is large, the ground state energy density is given by

$$
E_{\text {vacuum }}=\frac{e^{2}}{2} \min _{n \in \mathbf{Z}}|t-n|^{2} .
$$

Therefore, if $t$ is away from integer points, the contribution from large value of $\sigma$ is suppressed in the infrared limit $e^{2} \rightarrow \infty$. On the other hand, as $t \rightarrow 0$, the potential barrier for the $C$ branch disappears and $\sigma$ can grow indefinitely large. This causes sigma-model amplitudes to diverge in the limit. To separate the singular part and the regular part of the $\sigma$ model functional integral, we find it useful to divide the Riemann surface $\mathcal{S}$ into the $C$ domain $U_{C}$ where $\sigma$ is large and the $H$ domain $U_{H}$ where it is small. For definiteness, we introduce a cutoff parameter $\sigma_{*}$ and define these regions and their boundary $\gamma$ as

$$
\begin{gathered}
U_{C}=\left\{z \in \mathcal{S}:|\sigma(z)|>\sigma_{*}\right\}, \\
U_{H}=\left\{z \in \mathcal{S}:|\sigma(z)|<\sigma_{*}\right\}, \\
\gamma=\left\{z \in \mathcal{S}:|\sigma(z)|=\sigma_{*}\right\} .
\end{gathered}
$$

We would like to stress that both the $C$ and $H$ domains are parts of the Higgs branch theory, as already discussed. The functional integral can then be performed in the following steps:

(1) Integral over $\sigma$ with $|\sigma|>\sigma_{*}$ and the rest of the fields in $U_{C}$. Because of the potential (3.16), the chiral multiplet fields $a_{i}, b_{i}$ are suppressed. This domain describes topological string localized at the conifold singularity.

(2) Integral over $\sigma$ with $|\sigma|<\sigma_{*}$ and the rest of the fields in $U_{H}$. Because of the cutoff, the functional integral does not generate singularities at $t=0$. This domain describes string propagating in the smooth part of the conifold.

(3) Integral over location of the boundary $\gamma$ of the two domains.

Let us discuss contributions from each integral separately. We will discuss (3) first. 


\subsection{Integral over $\gamma$}

The location and shape of the boundary $\gamma$ can be regarded as collective coordinates of $\sigma$. The task of identifying these collective coordinates is simplified by the localization property of topological sigma-model functional integral. We note that the gauge invariant field strengh $\Sigma$ of the vector multiplet defined in (3.24) is a twisted chiral superfield, and the roles of the $\mathrm{A}$ and $\mathrm{B}$ twists are exchanged when acting on the vector multiplet fields. In the topological twisting in question, both $\partial_{z} \sigma$ and $\partial_{\bar{z}} \sigma$ are BRST trivial. Therefore the functional integral over $\sigma$ on a Riemann surface of fixed moduli reduces to a finite dimensional integral over constant mode of $\sigma$. This means that the entire Riemann surface is either in the $C$ domain or in the $H$ domain.

The situation is more subtle in the topological string where we integrate over the moduli space of Riemann surfaces. As explained in [7] and clarified further in [14], the scalar field $\sigma$ can be non-constant if we take the limit in the moduli space where the worldsheet is infintely elongated along the direction in which $\sigma$ is varying so that variation of $\sigma$ per unit worldsheet length goes to zero. This means that the boundary $\gamma$ between the $C$ and $H$ domains can emerge if and only if the worldsheet has a long cylindrical region and $\gamma$ cuts through the cylinder, as shown in Figure 7.

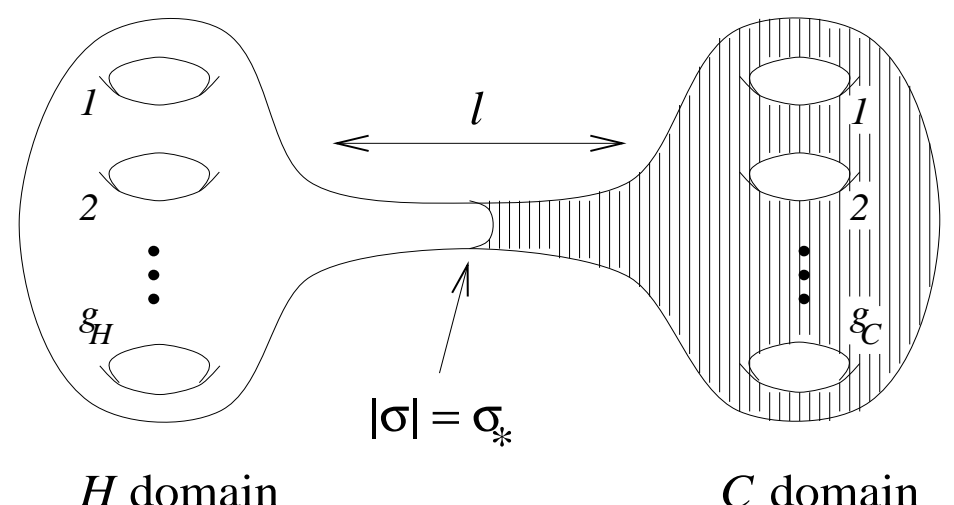

Figure 7: A worldsheet can be separated into the two domains across a long cylinder.

Let us introduce coordinates $(x, y)$ on the cylindrical region of the worldsheet, where $x$ is a periodic coordinate around the cylinder, $x \sim x+2 \pi$, and $y$ take value in the interval $0 \leq y \leq l$ for some large $l$. The cylinder is attached to the rest of the Riemann surface at the two ends, $y=0$ and $l$. Suppose the boundary $\gamma$ is located at $y=y_{0}$; the $H$ domain on the left $y<y_{0}$ and the $C$ domain is on the right $y>y_{0}$. By the topological reduction, the 
$\sigma$ field is varying only along the $y$ direction and stays constant in the $x$ direction. Namely the vector multilet field reduces to a quantum mechanical model with $y$ playing the role of imaginary time.

On the cylinder, we are performing the functional integral with the constraints that $|\sigma|=\sigma_{*}$ at $y=y_{0}$. As discussed in the above, the localization of the topological B-model for the $\sigma$ field implies that, along the boundary $\gamma$, the functional integral over $\sigma$ reduces to a finite dimensional integral over constant value of $\sigma$. We thus have

$$
\sigma(z)=\sigma_{0} \quad \text { at } \quad y=y_{0}
$$

where

$$
\sigma_{0}=\sigma_{*} e^{i \varphi}
$$

for some fixed $\varphi$. Of course the topological sigma model will not fix the phase of $\sigma_{0}$ and thus we have to integrate over $\varphi$. Since the topological string is B-twisted in the $C$ domain, the functional integral in the $C$ domain on the right of $\gamma$ gives an amplitude which depends holomorphicaly on $\sigma_{0}$. The amplitude from the $H$ domain on the left will turn out to be independent of $\sigma_{0}$.

In the original functional integral of the linear sigma-model, the value of $\sigma$ at $y=y_{0}$ is arbitrary. The constraint $|\sigma|=\sigma_{*}$ then transforms the integral over $|\sigma|$ at $y_{0}$ into an integral over the location $y_{0}$ of the boundary $\gamma$ along the cylinder. Let us evaluate the Jacobian for this change of funcational integral variables $(\sigma, \bar{\sigma}) \rightarrow\left(y_{0}, \varphi\right)$. We can write

$$
d \sigma\left(y_{0}\right) \wedge d \bar{\sigma}\left(y_{0}\right)=d \sigma_{0} \wedge d y_{0} \cdot \frac{d \bar{\sigma}}{d y}\left(y_{0}\right)
$$

The operator $d \bar{\sigma} / d y$ is a marginal operator which shifts the boundary condition $\sigma=\sigma_{0} \rightarrow$ $\sigma_{0}+\epsilon$. To see this note that $d \bar{\sigma} / d y$ is the momentum conjugate to $\sigma$ (viewing $y$ as the time direction along the cylinder) and so it corresponds to $\partial / \partial \sigma$. Therefore we can make the change of the functional integral variables as

$$
d \sigma\left(y_{0}\right) \wedge d \bar{\sigma}\left(y_{0}\right) \sim d y_{0} \wedge d \sigma_{0} \cdot \frac{\partial}{\partial \sigma_{0}}
$$

Here the derivative operator on the right-hand side acts on the amplitudes computed in the $C$ and $H$ branches. We still have to integrate over the localization moduli for the B-model, which means the phase of the $\sigma$ field. Thus we find that the integral over $\gamma$ effectively turns into

$$
d y_{0} \oint d \sigma_{0} \cdot \frac{\partial}{\partial \sigma_{0}}
$$


where the contour integral is over $|\sigma|=\sigma_{*}$. The derivative will act on the path integral on the Coulomb branch with the boundary condition $\sigma=\sigma_{0}$ on $\gamma$. Since the topological Bmodel amplitude is holomorphic in $\sigma_{0}$, the precise location of the above integral is irrelevant as long as it circles the origin, where the topological amplitude may have singularities. The integral over $d y_{0}$, together with the overall length $l$ of the cylinder, is exactly what one expects for the integration over division of the cyclinder to two regions, as one would need for the interpretation of $H$ and $C$ regions as independent string theories, whose amplitudes are evaluated by integrating over moduli spaces of Riemann surfaces with boundaries.

\subsection{Contribution from the $C$ domain}

The $C$ domain describes topological string at the conifold singularity. Since the chiral multiplet fields are massive, we can integrate them out. This gives rise to a linear dilaton coupling for the vector multiplet, which changes the measure for $\sigma$ [4]. In addition, we have the twisted superpotential (3.22). We will utilize this description to compute amplitudes in the $U_{C}$ domain.

A topological string amplitude is given by an integral of a topological sigma-model correlation function over the moduli space of Riemann surface. For example, the topological string partition function at $g$ loop is defined by (4.1). When the worldsheet is divided into the $C$ and $H$ domains, the $(6 g-6)$-dimensional moduli space of $\mathcal{M}_{g}$ becomes locally a product of the moduli spaces of $U_{C}$ and $U_{H}$. The simplest cases are when $\mathcal{S}=U_{C}$ or $U_{H}$, namely when the entire Riemann surface is either in the $C$ domain or in the $H$ domain. The contribution from the case $\mathcal{S}=U_{C}$ is denoted by $\mathcal{F}_{g, 0}^{(C)}$, where 0 refers to the fact that that is no boundary. We will compute this in (b) below. There is also a possibility that the entire Riemann surface is in the $H$ domain. This will be discussed in the next subsection.

Now let us consider the case when the boundary $\gamma$ has one connected component, which separate the surface into $U_{C}$ and $U_{H}$. Suppose $U_{C}$ has $g_{C}$ handles and $U_{H}$ has $g_{H}$ handles so that $g=g_{C}+g_{H}$. Among $(6 g-6)$ Bertrami differentials on $\mathcal{S}$, we can choose $\left(6 g_{C}-6\right)$ to be localized in $U_{C}$ and $\left(6 g_{H}-6\right)$ to be localized in $U_{H}$. The remaining six moduli parameter are associated to the long cylindrical region connecting $U_{C}$ and $U_{H}$ discussed in the last subsection. Among them, two are associated to the location of an end point of the cylinder in $U_{C}$, two are associated to the other end point of the cylinder in $U_{H}$, and the remaining two describe the length $L$ and the twist of the cylinder. If the surface $\mathcal{S}$ is separated into the two domains $U_{C}$ and $U_{H}$, the twist parameter becomes irrelevant 
since we can twist the cylinder along the boundary without causing any change to $U_{C}$ or $U_{H}$. This is compensated by the fact that the location $y_{0}$ of the boundary $\gamma$ becomes an additional moduli parameter, so the total number of moduli remains the same. We find $\left(6 g_{C}-3\right)$ moduli parameters for $U_{C}$ and $\left(6 g_{H}-3\right)$ moduli parameters for $U_{H}$.

One can also see that the insertions of $G_{L}^{-}$and $G_{R}^{-}$in (4.1) are distributed in the $C$ and $H$ domains in such a way that we obtain the correct measures for the topological string amplitudes on $U_{C}$ and $U_{H}$ with the boundary $\gamma$. This is obvious for the moduli whose Beltrami differentials are localized in either $U_{C}$ or $U_{H}$. The only slightly nontrivial ones are the insertions of $G_{L}^{-}$and $G_{R}^{-}$associated to the length and twist of the cylinder. These operators are integrated along the $x$ direction as $\int_{0}^{2 \pi} d x G_{L}^{-}(x, y) \int_{0}^{2 \pi} d x G_{R}^{-}(x, y)$ at some fixed $y$. We can move one of them to the $H$ domain $y<y_{0}$ and the other one to the $C$ domain $y>y_{0}$, and use them to give the correct measures for the extra moduli.

Let us denote the amplitudes on $U_{C}$ and $U_{H}$ separated by the single boundary $\gamma$ by where $\mathcal{F}_{g_{C}, 1}^{(C)}$ and $\mathcal{F}_{g_{H}, 1}^{(H)}$. Taking into account the Jacobian factor (4.7), their contribution to the topological string amplitude is

$$
\left(\mathcal{F}_{g}\right)_{\# \gamma=1}=\sum_{g_{C}+g_{H}=g} \Delta \mathcal{F}_{g_{C}, 1}^{(C)} \cdot \mathcal{F}_{g_{H}, 1}^{(H)}
$$

where

$$
\Delta \mathcal{F}_{g_{C}, 1}^{(C)}=\oint d \sigma_{0} \frac{\partial \mathcal{F}_{g_{C}, 1}^{(C)}}{\partial \sigma_{0}}
$$

computes the monodromy of $\mathcal{F}_{g_{C}, 1}^{(C)}$ around $\sigma_{0}=0$. This comes from doing path integral over $\gamma$ leading to (4.8) as discuused before. Clearly the contribution vanishes if $\mathcal{F}_{g_{C}, 1}^{(C)}$ is a single-valued function of $\sigma_{0}$. This is the case for $g_{C} \geq 1$ since the amplitude has a well-defined expression as a correlation function of $\left(6 g_{C}-3\right)$ supercurrents $G^{-}$integrated over the moduli space of surface with $g_{C}$ handles and one hole. The only case when the amplitude could potentially have a nontrivial monodromy around $\sigma_{0}=0$ is when $g_{C}=0$, i.e. when the $C$ domain has the topology of the disk. Note that $6 g_{C}-3=-3$ in this case, and the amplitude $\mathcal{F}_{0,1}^{(C)}$ itself is not defined as a correlation function of the A-model. To compute it, we consider a well-defined correlation function such as $\partial^{2} \mathcal{F}_{0,1}^{(C)} / \partial \sigma_{0} \partial t$ and integrate it with respect to $t$ and $\sigma_{0}$. Actually it turns out that $\partial \mathcal{F}_{0,1}^{(C)} / \partial \sigma_{0}$ is already well defined, and we use it to compute $\Delta \mathcal{F}_{0,1}^{(C)}$. We will show later that the disk amplitude $\mathcal{F}_{0,1}^{(C)}$ indeed has a non-trivial monodromy around $\sigma_{0}=0$. To summarize we have shown that, when the Riemann surface is separated into one $C$ domain and one $H$ domain, the only 
contribution to the topological string amplitude comes from the case when the $C$ domain has a topology of disk, as shown in Figure 8.

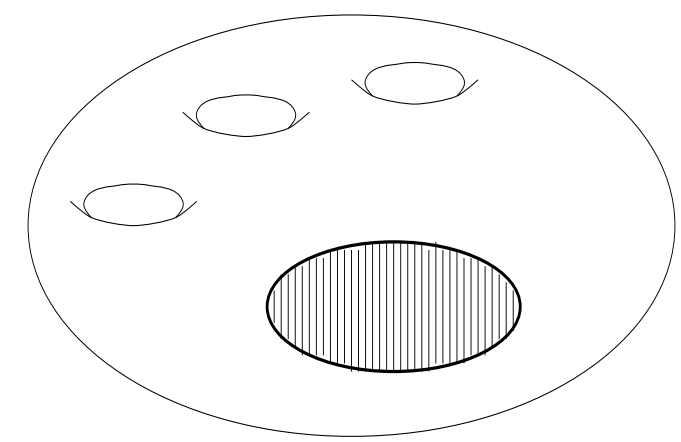

Figure 8: $A$ worldsheet with both $H$ and $C$ domains contributes to the topological string amplitude only when every $C$ domain has the topology of the disk.

It is straightforward to generalize this consideration to the case when $\gamma$ has several connected components. The Jacobian factor (4.8) appears on each boundary component. We then perform the angular integral $\oint d \sigma_{0}$ for each boundary. We can show that the amplitude is non-zero only when every connected component of $U_{C}$ has topology of disk. Suppose $U_{C}$ has a connected component with $g$ handles and $h$ boundaries. The topological string amplitude computed on that component with the Dirichlet boundary condition is well-defined if $6 g+3 h-6 \geq 1$. Therefore, if $h \geq 1$, there are only two cases when the amplitude can have monodromy; $h=1$ or 2 with $g=0$, i.e. it is either a disk or an annulus. Since the annulus has two boundaries, its contribution to the topological string amplitude is

$$
\oint d \sigma_{0} \oint d \sigma_{0}^{\prime} \frac{\partial^{2} \mathcal{F}_{0,2}^{(C)}}{\partial \sigma_{0} \partial \sigma_{0}^{\prime}},
$$

where $\mathcal{F}_{0,2}^{(C)}$ is the annulus amplitude with the boundary conditions $\sigma=\sigma_{0}$ on one boundary and $\sigma=\sigma_{0}^{\prime}$ on the other. Although $\mathcal{F}_{0,2}^{(C)}$ may have a monodromy, its derivative $\partial \mathcal{F}_{0,2}^{(C)} / \partial \sigma_{0}^{\prime}$ is a single-valued holomorphic function of $\sigma_{0}$. This means that the result of the $\sigma_{0}$ integral for fixed $\sigma_{0}^{\prime}$ in (4.11) gives zero. Therefore the mixed phase with the $C$ branch with the topology of the annulus, as shown in Figure 9, does not contribute to the topological string amplitude. We have found that the $C$ domain must be a disjoint union of disks. 


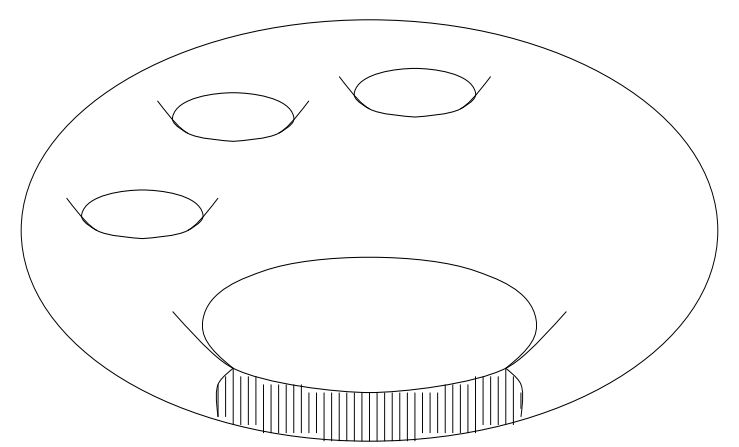

Figure 9: The partition function of the $C$ domain with the topology of the annulus can have a non-zero monodromy as a function of $\sigma_{0}$ and $\sigma_{0}^{\prime}$, which specify the Dirichlet boundary conditions on $\sigma(z)$. However the amplitude is annihilated by $\oint d \sigma_{0} \oint d \sigma_{0}^{\prime} \partial_{\sigma_{0}} \partial_{\sigma_{0}^{\prime}}$ and therefore does not contribute to the topological string amplitude.

We conclude the $g$ loop topological string amplitude is expressed as

$$
\mathcal{F}_{g}=\mathcal{F}_{g, 0}^{(C)}+\sum_{h=1}^{\infty}\left(\Delta \mathcal{F}_{0,1}^{(C)}\right)^{h} \cdot \mathcal{F}_{g, h}^{(H)}
$$

where $\Delta \mathcal{F}_{0,1}^{(C)}$ is the monodromy of the disk amplitude and $\mathcal{F}_{g, h}^{(H)}$ is the contribution from the $H$ domain with $g$ handles and $h$ holes.

Let us compute $\mathcal{F}_{0,1}^{(C)}$ and $\mathcal{F}_{g, 0}^{(C)}$.

(a) $\mathcal{F}_{0,1}^{(C)}$

As discussed above, in order to evaluate the contribution of the disk amplitude we need to compute

$$
\Delta \mathcal{F}_{0,1}^{(C)}=\oint d \sigma_{0} \frac{\partial \mathcal{F}_{0,1}^{(C)}}{\partial \sigma_{0}}
$$

The amplitude $\mathcal{F}_{0,1}^{(C)}$ is evaluated in the $C$ domain of disk topology with the Dirichlet boundary condition $\sigma=\sigma_{0}$. As noted in 30,31, 32, $\mathcal{F}_{0,1}^{(C)}$ is given by the integral of the top holomorphic form bounding the brane. (In the case of Calabi-Yau threefold this corresponds to a 2-cycle in Calabi-Yau with the holomoprhic 3-form integrated over a 3 -chain with boundary being the 2-cycle.) In the $C$ domain, we effectively have a LandauGinzburg theory with superpotential

$$
W=t \Sigma
$$


$\Sigma$ is the twisted chiral superfield with $\sigma$ as its lowest component. As discussed in [33] for an $\mathcal{N}=2$ Landau-Ginzburg model, the top holomorphic form is given by

$$
\prod_{i} d \Phi_{i} e^{W\left(\Phi_{i}\right)}
$$

In the case at hand, we have a single chiral field $\sigma$. However the measure in the field space is not $d \sigma$ but rather

$$
\frac{d \sigma}{\sigma^{2}}
$$

as noted in [4]. This arises by integrating over the chiral fields $A_{i}, B_{i}$ which are massive in the $C$ domain, which leads to a dilaton which translates to the above change in measure. In the $C$ domain, the brane corresponds to the 0 -cycle specified by the point $\sigma=\sigma_{0}$ and thus the disk amplitude is an integral over a chain with $\sigma_{0}$ as its boundary, i.e.

$$
\mathcal{F}_{0,1}^{(C)}\left(\sigma_{0}\right)=\int^{\sigma_{0}} \frac{d \sigma}{\sigma^{2}} e^{t \sigma}
$$

Thus we have

$$
\Delta \mathcal{F}_{0,1}^{(C)}=\oint d \sigma_{0} \frac{\partial \mathcal{F}_{0,1}^{(C)}}{\partial \sigma_{0}}=\oint d \sigma_{0} \frac{e^{t \sigma_{0}}}{\sigma_{0}^{2}} \propto t,
$$

which is what we wanted to show. Thus each disk in the $C$ domain contributes $t=N \lambda_{s}$ to the $H$ branch amplitude. This is exactly what we need in order for the string amplitude to reproduce the 't Hooft expansion (1.2).

(b) $\mathcal{F}_{g, 0}^{(C)}$

We now evaluate the amplitude when the entire Riemann surface is in the $C$ domain. There is an equivalent description of the $C$ domain in terms of another Landau-Ginzburg theory, which can be motivated by trying to make the measure in (4.13) be a more standard one. If we define the chiral field $X$ by

$$
X=\Sigma^{-1},
$$

the superpotential is expressed as

$$
W=t X^{-1},
$$

and the field measure changes to

$$
d x=-\frac{d \sigma}{\sigma^{2}}
$$


Thus, in terms of $X$, the linear dilaton is turned off and we have an ordinary LandauGinzburg theory. In fact this model has been proposed in [34] as being related to the $c=1$ bosonic string with a target circle at self-dual radius. This follows from the fact that the Landau-Ginzburg model with the superpotential (4.14) is equivalent to the $S L(2, R) / U(1)$ coset conformal field theory at level 3 , which in turn is related to the $c=1$ bosonic string. Originally this equivalence was motivated by an "analytic contiuation" to $k=-3$ of the well-established equivalence between the Landau-Ginzburg mode with the superpotential $W=X^{k+2}$ and the minimal $\mathcal{N}=2$ conformal field theory, which can be realized as the $S U(2) / U(1)$ coset model at level $k$. More recently, this equivalence was proven directly using mirror symmetry in [35]. In particular, the topological B-model with the superpotential (4.14) is mapped to the topological A-model on $S L(2, R) / U(1)$ at level 3 . In the $S L(2, R) / U(1)$ coset model, the topological string amplitude $\mathcal{F}_{g}(S L(2, R) / U(1))$ at genus $g$ has been computed 36] with the result,

$$
\mathcal{F}_{g}(S L(2, R) / U(1))=\frac{\chi\left(\mathcal{M}_{g}\right)}{t^{2 g-2}}
$$

where $\chi\left(\mathcal{M}_{g}\right)$ is the Euler characteristic of $\mathcal{M}_{g}$. More explicitly,

$$
\mathcal{F}_{g}(S L(2, R) / U(1))=\frac{B_{2 g}}{2 g(2 g-2) t^{2 g-2}}
$$

for $g \geq 2$, where $B_{2 g}$ is the $2 g$-th Bernoulli number. This can also be interpreted as the partition function of the $c=1$ bosonic string on a self-dual circle [37]. From the above chain of equivalences, we find that (4.15) gives the contribution to the topological string amplitude when the whole Riemann surface is in the $C$ domain.

To conclude, we have

$$
\mathcal{F}_{g, 0}^{(C)}=\frac{B_{2 g}}{2 g(2 g-2) t^{2 g-2}},
$$

for $g \geq 2$. The topological string amplitudes at $g=0$ and 1 are somewhat special. For $g=0$, we have

$$
\mathcal{F}_{0,0}^{(C)}=\frac{1}{2} t^{2} \log t+Q(t)
$$

where $Q(t)$ is a quadratic polynomial in $t$. The easiest way to see that is to note that $\partial \mathcal{F}_{0,0}^{(C)} / \partial t$ is a period integral given by

$$
\partial \mathcal{F}_{0,0}^{(C)} / \partial t=\int d \sigma \sigma^{-2} e^{t \sigma}
$$


Taking two more derivatives with respect to $t$ allows one to do the integral and one obtains $\partial^{3} \mathcal{F}_{0,0}^{(C)} / \partial t^{3}=t^{-1}$ which can be integrated to find the above result. The genus 1 amplitude is

$$
\mathcal{F}_{1,0}^{(C)}=-\frac{1}{12} \log t
$$

We find that (4.16), (4.17) and (4.19) reproduce the nonperturbative terms (2.12) in the Chern-Simons theory amplitude that are not captured in the 't Hooft expansion.

As an aside, we would like to point out that the above results also provide another insight into the claim that the Landau-Ginzburg B-model with $W=t X^{-1}$ is equivalent to the B-model conformal theory on the deformed conifold [19]. This follows from the fact that the most singular contribution to A-model amplitudes on resolved conifold when $t \rightarrow 0$ should come from the $C$ domain which opens up at that point, giving rise to the results in the above paragraphs. On the other hand in this limit, the mirror symmetry maps the resolved conifold to the deformed conifold. This explains the equivalence between the B-model on the deformed conifold and the above Landau-Ginzburg B-model.

Thus we have a number of equivalences:

- The topological Landau-Ginzburg B-model with the superpotential $W=t \Sigma$ with the non-standard measure $d \sigma / \sigma^{2}$.

- The topological Landau-Ginzburg B-model with the superpotential $W=t X^{-1}$ with the standard measure.

- The $t \rightarrow 0$ limit of the topological A-model on the resolved conifold.

- The $S L(2, R) / U(1)$ coset A-model.

- The $c=1$ string theory at the self-dual radius.

Moreover the contribution from this piece is precisely the part that the perturbative ChernSimons theory lacks from the exact results.

Note that these equivalences also gives rise to an a priori formula relating the volume of $U(N)$ to the Euler characteristic $\chi\left(\mathcal{M}_{g}\right)$ of the moduli space of genus $g$ Riemann surfaces as

$$
\log (\operatorname{vol}(U(N)))=-\sum_{g} \frac{\chi\left(\mathcal{M}_{g}\right)}{N^{2 g-2}}
$$

with an appropriate interpretion for $g=0$ and 1 terms. To our knowledge, this intriguing formula has not been noted in any matrix model or topological string literature. 


\subsection{Contribution from the $H$ domain}

In the $H$ domain, the functional integral over $\sigma$ is cutoff at $|\sigma|<\sigma_{*}$ and therefore is regular in the conifold limit. We can regard this domain as describing topological string propagating in the smooth part of the conifold. As explained in section 2, the smooth resolution is a local operation at the conifold singularity, so we expect that amplitudes computed in the $H$ domain to be independent of the Kähler moduli $t$. In fact, since the $U(1)$ gauge symmetry is spontaneously broken in the $H$ domain, the field strength $F$ is zero and there is no contribution from the theta term, $i \frac{\theta}{2 \pi} F$. By holomorphy in $t$, we conclude that the amplitude in the $H$ domian does not depend on $t$. Thus we will evaluate the contribution from the $H$ domain right at the conifold limit $t=0$.

In the $C$ domain, the chiral multilet fields $a_{i}, b_{i}$ are suppressed due to the potential (3.16) with $|\sigma|>\sigma_{*}$. Viewed from the $H$ domain, we have the Dirichlet condition $a_{i}, b_{i}=0$ at the boundary. Namely the chiral multiplet fields in the $H$ domain is ending on the conifold singularity. To evaluate the amplitude, it is useful to regard the conifold as the $\mu \rightarrow 0$ limit of the deformed conifold geometry (3.3). Since the deformation parameter $\mu$ is BRST trivial in the topological A-model, the amplitude is independent of $\mu$. Thus we can turn on $\mu$ without changing the amplitude. To do this, we need to use $y_{i}$ variables which are related to $a_{i}, b_{i}$ by (3.13) when $\mu=0$. When $\mu>0$, the deformed conifold is the cotangent bundle over $S^{3}$, as we saw in section 2 , and the natural boundary condition for the A-model is that the open string ends on the base $S^{3}$. In the phase space coordinate (3.4), the conditions are $p_{i}=0$, which correspond in the $y_{i}$ coordinates to

$$
y_{1}=\bar{y}_{2}, \quad y_{3}=-\bar{y}_{4} .
$$

In the limit $\mu \rightarrow 0$, these conditions together with the equation for the conifold geometry (3.8) imply $y_{i}=0$ and reduces to the description in terms of $a_{i}, b_{i}$ with the fully Dirichlet

condition on them. Since the A-model amplitude is independent of $\mu, \mathcal{F}_{g, h}^{(H)}$ should be given by the topological string amplitude with $\mathrm{D}$ brane wrapping on the base $S^{3}$ of $T^{*} S^{3}$. According to [7], this is nothing but the sum over Feynman diagrams of the Chern-Simons gauge theory on $S^{3}$ denoted by $F_{g, h}$ in section 3 . Here we are obtaining the strictly zero size limit of $S^{3}$. However when we deform $S^{3}$ to finite size, the topological A-model amplitudes do not change, and the only allowed D-brane in the geometry is the one corresponding to the branes wrapping $S^{3}$. Thus, smoothing out the geometry gives the uniquely allowed branes, yielding the description of $U(N)$ Chern-Simons on $S^{3}$. 
As we mentioned in the last subsection, there is also the possibility that the entire Riemann surface is in the $H$ domain. This would describe the closed topological string on the conifold with finite size $S^{3}$, and its amplitudes is trivial since there is no two-cycles which is needed for worldsheet instantons.5 This is consistent with the expectation that the closed string degrees of freedom should have been decoupled in the gauge theory side.

\subsection{Summary}

We have found that, by summing over configurations of the $C$ and $H$ domains, the $g$ loop topological string amplitude for $g \geq 2$ is given by

$$
\mathcal{F}_{g}=\frac{B_{2 g}}{2 g(2 g-2) t^{2 g-2}}+\sum_{h=1}^{\infty} t^{h} F_{g, h} .
$$

The sum over $h$ in the second term reproduces the 't Hooft expansion of the Chern-Simons gauge theory, and the coefficients $F_{g, h}$ are computed using ribbon graphs with $g$ handles and $h$ holes. On the other hand, the first term comes from the situation when the entire Riemann surface is in the $C$ domain. This term is missing in the 't Hooft expansion, as discussed in section 2 .

The formulae for $g=0$ and 1 are given by

$$
\begin{aligned}
& \mathcal{F}_{g=0}=\frac{t^{2}}{2} \log t+Q(t)+\sum_{h=1}^{\infty} t^{h} F_{g=0, h}, \\
& \mathcal{F}_{g=1}=-\frac{1}{12} \log t+\sum_{h=1}^{\infty} t^{h} F_{g=1, h},
\end{aligned}
$$

where $Q(t)$ is some quadratic polynomial of $t$. Combining (4.22) and (4.23) together and coverting the variables as $t=g_{\mathrm{YM}}^{2} N$ and $\lambda_{s}=g_{\mathrm{YM}}^{2}$, the topological string amplitude to all order in the perturbative expansion is given by

$$
\begin{aligned}
\mathcal{F}= & \sum_{g=0}^{\infty} g_{\mathrm{YM}}^{2 g-2} \mathcal{F}_{g}^{(C)} \\
= & \frac{N^{2}}{2} \log \left(g_{\mathrm{YM}}^{2} N\right)-\frac{1}{12} \log \left(g_{\mathrm{YM}}^{2} N\right)+\sum_{g=2}^{\infty} \frac{1}{N^{2 g-2}} \frac{B_{2 g}}{2 g(2 g-2)} \\
& \quad+g_{\mathrm{YM}}^{-4} Q\left(g_{\mathrm{YM}}^{2} N\right) \\
& +\sum_{g=0}^{\infty} \sum_{h=1}^{\infty}\left(g_{\mathrm{YM}}\right)^{2 g+h-2} N^{h} F_{g, h} .
\end{aligned}
$$

5 Even degenerate instantons do not contribute in this case. 
The sum over $g$ and $h$ in the last term in the right-hand side reproduces the 't Hooft expansion of the Chern-Simons gauge theory. The non-perturbative conributions of the gauge theory are also in perfect agreement with the terms in the above coming from the case when the entire Riemann surface is in the Coulomb branch.

\section{5. $S O(N)$ and $S p(N)$ gauge groups}

It was conjectured in [38] that the Chern-Simons gauge theories on $S^{3}$ with $S O(N)$ and $S p(N)$ gauge groups are dual to the topological closed string theories on an orientifold of the resolved conifold, with the dictionary that

$$
\begin{aligned}
\lambda_{s} & =\frac{i}{k+c_{g}}, \\
t & =(N+a) \lambda_{s},
\end{aligned}
$$

with $c_{g}=N-2, a=-1$ for $S O(N)$ and $c_{g}=N+1, a=+1$ for $S p(N)$. It should be possible to extend the proof in this paper to these cases with the orientifolds.

In particular, this means that the identity (4.20) for $U(N)$ can be generalized and that there is a relation between the volumes of $S O(N)$ and $S p(N)$ gauge groups and virtual Euler characteristics on the moduli spaces of orientable and non-orientable Riemann surfaces. The volumes of these groups can be evaluated using the formulae in [24, 39]

$$
\begin{aligned}
\operatorname{vol}(S O(2 n+1)) & =\frac{2^{n+1}(2 \pi)^{n^{2}+n-\frac{1}{4}}}{(2 n-1) !(2 n-3) ! \cdots 3 ! 1 !} \\
\operatorname{vol}(S O(2 n)) & =\frac{\sqrt{2}(2 \pi)^{n^{2}}}{(2 n-3) !(2 n-5) ! \cdots 3 ! 1 !(n-1) !} \\
\operatorname{vol}(S p(2 n)) & =\frac{2^{-n}(2 \pi)^{n^{2}+n}}{(2 n-1) !(2 n-3) ! \cdots 3 ! 1 !}
\end{aligned}
$$

The factorials in the denominator of each formula is related to the exponents of the cor-

responding group. Note that the expressions for $\operatorname{vol}(S O(2 n))$ and $\operatorname{vol}(S O(2 n+1))$ are related to each other by analytic continuation in $n$. This can be shown by using

$$
\begin{aligned}
(2 n-3) !(2 n-5) ! \cdots 3 ! 1 !(n-1) ! \rightarrow & 2^{-n} \sqrt{\pi}(2 n-1) !(2 n-5) ! \cdots 3 ! 1 ! \\
& \left(n \rightarrow n+\frac{1}{2}\right) .
\end{aligned}
$$

The analyticity in $n$ is necessary in order for the large $n$ expansion to make sense. One can also check explicitly that these formulae agree with the $k \rightarrow \infty$ limit of the exact results of the $S O$ and $S p$ Chern-Simons gauge theory. 
Let us evaluate the large $n$ expansion of the logarithm of each of these volume formulae and compare with the virtual Euler characteristics of the moduli spaces. As pointed out in [38], the non-orientable Riemann surfaces with two cross caps do not contribute to the topological closed string amplitudes. Therefore topological amplitudes with the orientifold of the $S O(S p)$ types are equal to one-half of the amplitude without the orientifold plus (minus) contributions with one crosscap. This means that the product of the volume of the $S O$ and $S p$ groups should be related to the volume of the unitary group. To be precise, we need to take into account the shift in $N \rightarrow N+a$ in the expression for $t$ in (4.25). Using the volume formulae (4.26) and performing the analytic continuation $2 n \rightarrow 2 n-a$ to compensate for the shift, we find

$$
\begin{aligned}
& \operatorname{vol}(S O(2 n+1)) \cdot \operatorname{vol}(S p(2 n-1)) \\
& =\sqrt{\frac{4}{\pi}} \frac{(2 \pi)^{2 n^{2}+n}}{(2 n-1) !(2 n-2) ! \cdots 2 ! 1 !}
\end{aligned}
$$

Comparing this with (2.7), we find

$$
\operatorname{vol}(S O(2 n+1)) \cdot \operatorname{vol}(S p(2 n-1))=\sqrt{\frac{4}{\pi}} \operatorname{vol}(U(2 n)) .
$$

Thus, using (4.20), we can write

$$
\begin{aligned}
& \log (\operatorname{vol}(S O(2 n+1)))+\log (\operatorname{vol}(S p(2 n-1))) \\
& =-\sum_{g} \frac{\chi\left(\mathcal{M}_{g}\right)}{(2 n)^{2 g-2}} .
\end{aligned}
$$

As in the case of $U(N)$, the $g=0$ and 1 terms need to be interpreted appropriately.

Similarly the large $N$ duality predicts that the logarithm of the ratio of these volumes gives a generating function of the virtual Euler characteristic $\chi\left(\mathcal{M}_{g}^{1}\right)$ is the Euler characteristic of moduli space of genus $g$ with a single cross cap as,

$$
\begin{aligned}
& \log (\operatorname{vol}(S O(2 n+1)))-\log (\operatorname{vol}(S p(2 n-1))) \\
& =\log \left(\frac{2^{-\frac{1}{2}}(4 \pi)^{n+\frac{1}{2}}}{\Gamma\left(n+\frac{1}{2}\right)}\right)=-\sum_{g} \frac{\chi\left(\mathcal{M}_{g}^{1}\right)}{(2 n)^{2 g-1}} .
\end{aligned}
$$

Using the expansion of the Gamma function, we find

$$
\chi\left(\mathcal{M}_{g}^{1}\right)=\frac{\left(2^{2 g-2}-2^{-1}\right) B_{2 g}}{2 g(2 g-1)} .
$$


This is in full agreement with the recent computation of this quantity [40].

To summarize, we found

$$
\begin{aligned}
& \log (\operatorname{vol}(S O(2 n+1)))=-\frac{1}{2} \sum_{g}\left(\frac{\chi\left(\mathcal{M}_{g}\right)}{(2 n)^{2 g-2}}+\frac{\chi\left(\mathcal{M}_{g}^{1}\right)}{(2 n)^{2 g-1}}\right), \\
& \log (\operatorname{vol}(S p(2 n-1)))=-\frac{1}{2} \sum_{g}\left(\frac{\chi\left(\mathcal{M}_{g}\right)}{(2 n)^{2 g-2}}-\frac{\chi\left(\mathcal{M}_{g}^{1}\right)}{(2 n)^{2 g-1}}\right) .
\end{aligned}
$$

As predicted by the large $N$ duality, the volume factors in the $S O$ and $S p$ Chern-Simons gauge theory are related to the topological string computation for orientable and nonorientable worldsheets in the pure $C$ domain.

\section{Discussion}

We have proven that the topological closed string amplitude $\mathcal{F}$ on the resolved conifold geometry can be expressed as

$$
\mathcal{F}=\mathcal{F}_{\text {nonpert }}+\sum_{g=0}^{\infty} \sum_{h=1}^{\infty}\left(g_{\mathrm{YM}}\right)^{2 g+h-2} N^{h} F_{g, h}
$$

where $F_{g, h}$ is the Chern-Simons amplitudes on $S^{3}$ computed by using ribbon graphs with $g$ handles and $h$ holes. In the closed string theory, $F_{g, h}$ computed using genus $g$ Riemann surfaces on which there are $h$ disks in the $C$ phase. Seen from the $H$ phase, these disks in the $C$ phase are holes on the worldsheet, which introduce an open string sector. We showed that, when the $C$ and $H$ phases coexist, each $C$ domain must have the topology of the disk. Moreover we found that each disk in the $C$ domain gives the factor $t=g_{Y M}^{2} N$, precisely as prescribed for the 't Hooft expansion. In this way, the closed string computation reproduces the Chern-Simons perturbative expansion.

The extra term $\mathcal{F}_{\text {nonpert }}$ is given by a sum over Riemann surfaces which are in the pure $C$ phase. This term, which cannot be captured in the 't Hooft expansion of the gauge theory, has various interpretations. From the string theory point of view, they are:

- The topological string coupled to the topological Landau-Ginzburg model with the superpotential $W=t X^{-1}$.

- The topological string coupled to the $S L(2, R) / U(1)$ coset A-model.

- The $c=1$ topological string amplitude on a circle of self-dual radius. 
These compute the Euler characteristic $\chi\left(\mathcal{M}_{g}\right)$ of the moduli space of genus $g$ Riemann surfaces. From the gauge theory point of view, they are:

- The measure factor of the Chern-Simons gauge theory on $S^{3}$.

- The fractional instanton contribution to superpotential terms on D6 branes wrapping on the $S^{3}$ of the deformed conifold geometry.

These compute the volume of $U(N)$. This leads to the equality (4.20) relating $\chi\left(\mathcal{M}_{g}\right)$ to the volume of $U(N)$. All these pictures fit together to make the gauge theory/string theory equivalence complete.

In this paper, we have presented the proof of the large $N$ duality using the partition function of the Chern-Simons theory as an example. It is not difficult to apply our derivation to other physical observables, such as expectation values of Wilson loops, which compute knot and link invariants. The proof goes as follows. As shown in [8], the computation involving Wilson loops can be formulated in the language of topological open string theory by adding probe D branes on $T^{*} S^{3}$ wrapping on special Lagrangian submanifolds which are determined by the configuration of the loops on $S^{3}$. Turning on holonomies of the gauge fields on the probe branes, the partition function of the topological open string theory gives a generating function of the knot and link invariants.6 The large $N$ duality predicts that the generating function can be computed as a partition function of the topological string on the resolved conifold in the presence of the corresponding probe $\mathrm{D}$ branes. It was pointed out in [12 that, in order to fix the framing ambiguity of the loops, one needs to move the probe $\mathrm{D}$ branes off from the base $S^{3}$, adding mass to the open string stretched to the probe $\mathrm{D}$ branes and the ones wrapping on the $S^{3}$. It is then clear what the corresponding branes are in the string theory side since the small resolution only modifies the geometry near the conifold point.

Given this, we can prove the duality involving the Wilson loops as follows. We start from the topological closed string on the resolved conifold with these probe $\mathrm{D}$ branes. We note that only the $H$ domain of the worldsheet can end on the probe $\mathrm{D}$ branes since they are away from the conifold point. We can then run the same argument as we presented in section 4 and show that this reproduces the topological open string computation with

6 The generating function is a function of the gauge field holonomies on the probe branes and carries information about Wilson loops expectation values for arbitrary representations of $U(N)$ running around the loops. 
the $N$ D branes wrapping on the base $S^{3}$ as well as the probe branes. In this way, the partition function of the topological closed string theory with the probe D branes can be shown to reproduce the generating function of the knots and links. In this case, we do not get the extra contribution from worldsheets in the pure $C$ phase since we need the $D$ domain in order for the worldsheet to end on the probe branes.

The proof of the large $N$ duality was made possible by the linear sigma-model description which is useful when the 't Hooft coupling $t=g_{\mathrm{YM}}^{2} N$ is small. It is in some sense a "non-perturbative" completion of the non-linear sigma model. Namely, if we had considered strictly the non-linear sigma model, we would have concluded that the worldsheet theory becomes singular at $t \rightarrow 0$. In the linear sigma model description, some massive states become light and relevant as new degrees of freedom in the infrared limit. It is somewhat similar to how the non-perturbative string states become light in the target physics, and are important in understanding the target dynamics. It would be interesting to come up with a similar description for strings in $A d S$, which should enable us to prove the $A d S / C F T$ correspondence from the first principle. We need an analogue of the linear sigma model for $A d S$ which gives a useful description of the worldsheet when the curvature radius of $A d S$ vanishes.

\section{Acknowledgments}

We thank M. Aganagic, R. Dijkgraaf, M. Douglas, R. Gopakumar, Y. Hashimoto, S. Katz, M. Marino, A. Strominger, N. Warner and E. Witten for valuable discussions. H.O. would like to thank the hospitality of the theory group at Harvard University. C.V. would like to thank the hospitality of the YITP at Stony Brook.

The research of HO was supported in part by DOE grant DE-FG03-92-ER40701. The research of CV was supported in part by NSF grants PHY-9802709 and DMS-0074329. 


\section{References}

[1] G. 't Hooft, "A planar diagram theory for strong interactions," Nucl. Phys. B 72, 461 (1974).

[2] R. Gopakumar and C. Vafa, "On the gauge theory/geometry correspondence," Adv. Theor. Math. Phys. 3, 1415 (1999); arXiv: hep-th/9811131.

[3] M. B. Green and J. Polchinski, "Summing over world sheet boundaries," Phys. Lett. B 335, 377 (1994); arXiv:hep-th/9406012.

[4] E. Silverstein and E. Witten, "Criteria for conformal invariance of $(0,2)$ models," Nucl. Phys. B 444, 161 (1995); arXiv: hep-th/9503212.

[5] E. Witten, "Some comments on string dynamics," arXiv:hep-th/9507121.

[6] H. Ooguri and C. Vafa, "Two-Dimensional Black Hole and Singularities of CY Manifolds," Nucl. Phys. B 463, 55 (1996); arXiv: hep-th/9511164.

[7] E. Witten, "Chern-Simons gauge theory as a string theory," arXiv: hep-th/9207094.

[8] H. Ooguri and C. Vafa, "Knot invariants and topological strings," Nucl. Phys. B 577, 419 (2000); arXiv:hep-th/9912123.

[9] M. F. Labastida and M. Marino, "Polynomial invariants for torus knots and topological strings," Commun. Math. Phys. 217, 423 (2001); arXiv: hep-th/0004196.

[10] P. Ramadevi and T. Sarkar, "On link invariants and topological string amplitudes," Nucl. Phys. B 600, 487 (2001); arXiv: hep-th/0009188.

[11] J. M. Labastida, M. Marino and C. Vafa, "Knots, links and branes at large N," JHEP 0011, 007 (2000); arXiv: hep-th/0010102.

[12] M. Marino and C. Vafa, "Framed knots at large $N$," arXiv:hep-th/0108064.

[13] C. Vafa, "Superstrings and topological strings at large $N$," J. Math. Phys. 42, 2798 (2001); arXiv:hep-th/0008142.

[14] M. Bershadsky, S. Cecotti, H. Ooguri and C. Vafa, "Kodaira-Spencer theory of gravity and exact results for quantum string amplitudes," Commun. Math. Phys. 165, 311 (1994); arXiv:hep-th/9309140.

[15] M. Atiyah, J. M. Maldacena and C. Vafa, "An $M$ theory flop as a large $N$ duality," J. Math. Phys. 42, 3209 (2001); arXiv: hep-th/0011256.

[16] B. S. Acharya, "On realising $\mathcal{N}=1$ super Yang-Mills in M-theory," arXiv:hepth/0011089.

[17] M. Aganagic and C. Vafa, "Mirror symmetry and a $G_{2}$ flop," arXiv: hep-th/0105225.

[18] M. Atiyah and E. Witten, " $M$ theory dynamics on a manifold of $G_{2}$ holonomy," arXiv: hep-th/0107177.

[19] D. Ghoshal and C. Vafa, " $c=1$ string as the topological theory of the conifold," Nucl. Phys. B 453, 121 (1995); arXiv: hep-th/9506122.

[20] E. Witten, "Quantum field theory and the Jones polynomial," Comm. Math. Phys. 121, 351 (1989). 
[21] D. Freed and R. Gompf, "Computer calculation of Witten's three manifold invariant," Commun. Math. Phys. 141, 79 (1991).

[22] L. C. Jeffrey, "Chern-Simons-Witten invariants of Lens spaces and torus bundles, and the semiclassical approximation," Commun. Math. Phys. 147, 563 (1992).

[23] L. Rozansky, "A large $k$ asymptotics of Witten's invariant of Seifert manifolds," Commun. Math. Phys. 171, 279 (1995); arXiv: hep-th/9303099.

[24] I. G. Macdonald, "The volume of a compact Lie group," Invent. Math. 56, 93 (1980).

[25] G. Veneziano and S. Yankielowicz, "An effective Lagrangian for the pure $\mathcal{N}=1$ supersymmetric Yang-Mills theory," Phys. Lett. B 113, 231 (1982).

[26] E. Witten, "Phases of $\mathcal{N}=2$ theories in two dimensions," Nucl. Phys. B 403, 159 (1993); arXiv: hep-th/9301042.

[27] S. R. Coleman, "More about the massive Schwinger model," Annals Phys. 101, 239 (1976).

[28] E. Witten, "On the conformal field theory of the Higgs branch," JHEP 9707, 003 (1997); arXiv: hep-th/9707093.

[29] O. Aharony and M. Berkooz, "IR dynamics of $d=2, \mathcal{N}=(4,4)$ gauge theories and DLCQ of 'little string theories'," JHEP 9910, 030 (1999); arXiv:hep-th/9909101.

[30] S. Kachru, S. Katz, A. Lawrence and J. McGreevy, "Open string instantons and superpotentials," Phys. Rev. D62 026001 (2000); arXiv:hep-th/9912151.

[31] M. Aganagic and C. Vafa, "Mirror symmetry, D branes and counting holomorphic discs," arXiv: hep-th/0012041.

[32] M. Aganagic, A. Klemm and C. Vafa, "Disk instantons, mirror symmetry and the duality web," Z. Naturforsch. A 57, 1 (2002); arXiv: hep-th/0105045.

[33] K. Hori, A. Iqbal and C. Vafa, "D branes and mirror symmetry," arXiv:hepth/0005247.

[34] S. Mukhi and C. Vafa, "Two-dimensional black hole as a topological coset model of $c=1$ string theory," Nucl. Phys. B 407, 667 (1993); arXiv:hep-th/9301083.

[35] K. Hori and A. Kapustin, "Duality of the fermionic $2 d$ black hole and $\mathcal{N}=2$ Liouville theory as mirror symmetry," JHEP 0108, 045 (2001); arXiv: hep-th/0104202.

[36] E. Witten, "On the structure of the topological phase of two-dimensional gravity," Nucl. Phys. B 340, 281 (1990).

[37] J. Distler and C. Vafa, "A critical matrix model at $c=1$," Mod. Phys. Lett. A 6, 259 (1991).

[38] S. Sinha and C. Vafa, "SO and Sp Chern-Simons at large N," arXiv: hep-th/0012136.

[39] Y. Hashimoto, unpublished notes.

[40] I.P.Goulden, J.L. Harer, and D.M. Jackson, "A geometric parametrization for the virtual Euler characteristic of the moduli spaces of real and complex algebraic curves," Trans. Am. Math. Soc. 353, 4405 (2001). 